\title{
Dissipation Induced Instabilities
}

\author{
Anthony Bloch * \\ Department of Mathematics, Ohio State University \\ Columbus, OH 43210 \\ P.S.Krishnaprasad ${ }^{\dagger}$ \\ Department of Electrical Engineering and \\ Institute for Systems Research \\ University of Maryland, College Park, MD 20742 \\ Jerrold E. Marsden $\ddagger$ \\ Department of Mathematics \\ University of California, Berkeley, CA 94720 \\ Tudor S. Ratiu $\S$ \\ Department of Mathematics \\ University of California, Santa Cruz, CA 95064
}

August, 1990; revised December 27, 1993, reprinted June 3, 1996

Analyse Nonlineaire, Annales Institute H. Poincaré, 11, (1994), 37-90

\begin{abstract}
The main goal of this paper is to prove that if the energy-momentum (or energy-Casimir) method predicts formal instability of a relative equilibrium in a Hamiltonian system with symmetry, then with the addition of dissipation, the relative equilibrium becomes spectrally and hence linearly and nonlinearly unstable. The energy-momentum method assumes that one is in the context of a mechanical system with a given symmetry group. Our result assumes that the dissipation chosen does not destroy the conservation law associated with the given symmetry group - thus, we consider internal dissipation. This also includes the special case of systems with no symmetry and ordinary equilibria. The theorem is proved by combining the techniques of Chetaev, who proved instability theorems using a special Chetaev-Lyapunov function, with those of
\end{abstract}

\footnotetext{
${ }^{*}$ Research partially supported by the National Science Foundation grant DMS-90-02136, PYI grant DMS-91-57556, and AFOSR grant F49620-93-1-0037

${ }^{\dagger}$ Research partially supported by the AFOSR University Research Initiative Program under grants AFOSR-87-0073 and AFOSR-90-0105 and by the National Science Foundation's Engineering Research Centers Program NSFD CDR 8803012

${ }^{\ddagger}$ Research partially supported by, DOE contract DE-FG03-92ER-25129, a Fairchild Fellowship at Caltech, and the Fields Institute for Research in the Mathematical Sciences

${ }^{\S}$ Research partially supported by NSF Grant DMS 91-42613 and DOE contract DE-FG0392ER-25129
} 
Hahn, which enable one to strengthen the Chetaev results from Lyapunov instability to spectral instability. The main achievement is to strengthen Chetaev's methods to the context of the block diagonalization version of the energy momentum method given by Lewis, Marsden, Posbergh, and Simo. However, we also give the eigenvalue movement formulae of Krein, MacKay and others both in general and adapted to the context of the normal form of the linearized equations given by the block diagoanl form, as provided by the energy-momentum method. A number of specific examples, such as the rigid body with internal rotors, are provided to illustrate the results.

\section{Contents}

1 Introduction $\quad 2$

2 The Energy-Momentum Method $\quad 11$

3 The Chetaev Function and Lyapunov Instability 22

4 Instability of Relative Equilibria $\quad 30$

5 Dissipation-Induced Movement of Eigenvalues $\quad 34$

6 Examples $\quad 44$

\section{Introduction}

A central and time honored problem in mechanics is the determination of the stability of equilibria and relative equilibria of Hamiltonian systems. Of particular interest are the relative equilibria of simple mechanical systems with symmetry, that is, Lagrangian or Hamiltonian systems with energy of the form kinetic plus potential energy, and that are invariant under the canonical action of a group. Relative equilibria of such systems are solutions whose dynamic orbit coincides with a one parameter group orbit. When there is no group present, we have an equilibrium in the usual sense with zero velocity; a relative equilibrium, however can have nonzero velocity. When the group is the rotation group, a relative equilibrium is a uniformly rotating state.

The analysis of the stability of relative equilibria has a distinguished history and includes the stability of a rigid body rotating about one of its principal axes, the stability of rotating gravitating fluid masses and other rotating systems. (See for example, Riemann [1860], Routh [1877], Poincaré [1885, 1892, 1901], and Chandrasekhar [1977]).

Recently, two distinct but related systematic methods have been developed to analyze the stability of the relative equilibria of Hamiltonian systems. The first, the energy-Casimir method, goes back to Arnold [1966] and was developed and formalized in Holm, Marsden, Ratiu, and Weinstein [1985], Krishnaprasad and Marsden [1987] and related papers. While the analysis in this method often takes place in a 
linear Poisson reduced space, often the "body frame", and this is sometimes convenient, the method has a serious defect in that a lack of sufficient Casimir functions makes it inapplicable to examples such as geometrically exact rods, three dimensional ideal fluid mechanics, and some plasma systems.

This deficiency was overcome in a series of papers developing and applying the energy-momentum method; see Marsden, Simo, Lewis and Posbergh [1989], Simo, Posbergh and Marsden [1990, 1991], Lewis and Simo [1990], Simo, Lewis, and Marsden [1991], Lewis [1992], and Wang and Krishnaprasad [1992]. These techniques are based on the use of the Hamiltonian plus a conserved quantity. In the energymomentum method, the relevant combination is the augmented Hamiltonian. One can think of the energy momentum method as a synthesis of the ideas of Arnold for the group variables, and those of Routh and Smale for the internal variables. In fact, one of the bonuses of the method is the appearance of normal forms for the energy and the symplectic structure, which makes the method particularly powerful in applications.

The above techniques are designed for conservative systems. For these systems, but especially for dissipative systems, spectral methods pioneered by Lyapunov have also been powerful. In what follows, we shall elaborate on the relation with the above energy methods.

The key question that we address in this paper is: if the energy momentum method predicts formal instability, i.e., if the augmented energy has a critical point at which the second variation is not positive definite, is the system in some sense unstable? Such a result would demonstrate that the energy-momentum method gives sharp results. The main result of this paper is that this is indeed true if small dissipation, arising from a Rayleigh dissipation function, is added to the internal variables of a system. (Dissipation in the rotational variables will be considered in another publication.) In other words, we prove that

If a relative equilibrium of a Hamiltonian system with symmetry is formally unstable by the energy-momentum method, then it is linearly and nonlinearly unstable when a small amount of damping (dissipation) is added to the system.

Some special cases of, and commentaries about, the topics of the present paper were previously known. As we shall discuss below, one of the main early references for this topic is Chetaev [1961] and some results were already known to Thomson and Tait [1912]. See also Ziegler [1956], Haller [1992], and Sri Namachchivaya and Ariaratnam [1985]. The latter paper shows the effect of dissipation induced instabilities for rotating shafts, and contains a number of other interesting references.

Next, we outline how the program of the present paper is carried out. To do so, we first look at the case of ordinary equilibria. Specifically, consider an equilibrium point $z_{e}$ of a Hamiltonian vector field $X_{H}$ on a symplectic manifold $P$, so that $X_{H}\left(z_{e}\right)=0$ and $H$ has a critical point at $z_{e}$. Then the two standard methodologies for studying stability mentioned above are as follows: 
1. Energetics - determine if

$$
\delta^{2} H\left(z_{e}\right)=: \mathcal{Q}
$$

is a definite quadratic form (the Lagrange-Dirichlet criterion).

2. Spectral methods - determine if the spectrum of the linearized operator

$$
\mathbf{D} X_{H}\left(z_{e}\right)=: L
$$

is on the imaginary axis.

The energetics method can, via ideas from reduction, be applied to relative equilibria too and this is the basis of the energy-momentum method alluded to above and which we shall detail in $\S \mathbf{3}$. The spectral method can also be applied to relative equilibria since under reduction, a relative equilibrium becomes an equilibrium.

For general (not necessarily Hamiltonian) vector fields, the classical Lyapunov theorem states that if the spectrum of the linearized equations lies strictly in the left half plane, then the equilibrium is stable and even asymptotically stable (trajectories starting close to the equilibrium converge to it exponentially as $t \rightarrow \infty$ ). Also, if any eigenvalue is in the strict right half plane, the equilibrium is unstable. This result, however, cannot apply to the purely Hamiltonian case since the spectrum of $L$ is invariant under reflection in the real and imaginary coordinate axes. Thus, the only possible spectral configuration for a stable point of a Hamiltonian system is if the spectrum is on the imaginary axis.

The relation between (a) and (b) is, in general, complicated, but one can make some useful elementary observations.

\section{Remarks}

1 Definiteness of $\mathcal{Q}$ implies spectral stability (i.e., the spectrum of $L$ is on the imaginary axis). This is because spectral instability implies (linear and nonlinear) instability (Lyapunov's Theorem), while definiteness of $\mathcal{Q}$ implies stability (the Lagrange Dirichlet criterion).

2 Spectral stability need not imply stability, even linear stability. This is shown by the unstable linear system $\dot{q}=p, \dot{p}=0$ with a pair of eigenvalues at zero. Other resonant examples exhibit similar phenomena with nonzero eigenvalues.

3 If $\mathcal{Q}$ has odd index (an odd number of negative eigenvalues), then $L$ has a real positive eigenvalue. This is a special case of theorems of Chetaev [1961] and Oh [1987]. Indeed, in canonical coordinates, and identifying $\mathcal{Q}$ with its corresponding matrix, we have

$$
L=\mathbb{J} \mathcal{Q} .
$$

Thus, $\operatorname{det} L=\operatorname{det} \mathcal{Q}$ is negative. Since $\operatorname{det} L$ is the product of the eigenvalues of $L$ and they come in conjugate pairs, there must be at least one pair of real eigenvalues, and since the set of eigenvalues is invariant under reflection in the imaginary axis, there must be an odd number of positive real eigenvalues. 
4 If $P=T^{*} Q$ with the standard cotangent symplectic structure and if $H$ is of the form kinetic plus potential so that an equilibrium has the form $\left(q_{e}, 0\right)$, and if $\delta^{2} V\left(q_{e}\right)$ has nonzero (even or odd) index, then again $L$ must have real eigenvalues. This is because one can diagonalize $\delta^{2} V\left(q_{e}\right)$ with respect to the kinetic energy inner product, in which case the eigenvalues are evident. In this context, note that there are no gyroscopic forces.

To get more interesting effects than covered by the above examples, we consider gyroscopic systems; i.e., linear systems of the form

$$
M \ddot{q}+S \dot{q}+\Lambda q=0
$$

where $q \in \mathbb{R}^{n}, M$ is a positive definite symmetric $n \times n$ matrix, $S$ is skew, and $\Lambda$ is symmetric. This system is verified to be Hamiltonian with $p=M \dot{q}$, energy function

$$
H(q, p)=\frac{1}{2} p M^{-1} p+\frac{1}{2} q \Lambda q
$$

and the bracket

$$
\{F, K\}=\frac{\partial F}{\partial q^{i}} \frac{\partial K}{\partial p_{i}}-\frac{\partial K}{\partial q^{i}} \frac{\partial F}{\partial p_{i}}-S_{i j} \frac{\partial F}{\partial p_{i}} \frac{\partial K}{\partial p_{j}} .
$$

Systems of this form arise from simple mechanical systems via reduction; this form is in fact the normal form of the linearized equations when one has an abelian group. Of course, one can also consider linear systems of this type when gyroscopic forces are added ab initio, rather than being derived by reduction. Such systems arise in control theory, for example; see Bloch, Krishnaprasad, Marsden, and Sanchez [1991] and Wang and Krishnaprasad [1992].

If the index of $V$ is even (see Remark 3) one can get situations where $\delta^{2} H$ is indefinite and yet spectrally stable. Roughly, this is a situation that is capable of undergoing a Hamiltonian Hopf bifurcation. One of the simplest systems in which this occurs is in the linearized equations about a special relative equilibrium, called the "cowboy" solution, of the double spherical pendulum; see Marsden and Scheurle [1993] and $\S 6$ below. Another example arises from certain solutions of the heavy top equations as studied in Lewis, Ratiu, Simo and Marsden [1992]. Other examples are given in $\S$. One of our first main results is the following:

Theorem 1.1 Dissipation induced instabilities - abelian case Under the above conditions, if we modify (1) to

$$
M \ddot{q}+(S+\epsilon R) \dot{q}+\Lambda q=0
$$

for small $\epsilon>0$, where $R$ is symmetric and positive definite, then the perturbed linearized equations

$$
\dot{z}=L_{\epsilon} z,
$$

where $z=(q, p)$ are spectrally unstable, i.e., at least one pair of eigenvalues of $L_{\epsilon}$ is in the right half plane. 
This result builds on basic work of Thomson and Tait [1912], Chetaev [1961], and Hahn [1967]. The argument proceeds in two steps.

\section{Step 1 Construct the Chetaev function}

$$
W(q, p)=H(q, p)+\beta M^{-1} p \cdot(\Lambda q)
$$

for small $\beta$ and use this to prove Lyapunov instability.

This function has the key property that for $\beta$ small enough, $W$ has the same index as $H$, yet $\dot{W}$ is negative definite, where the overdot is taken in the dynamics of (4). This is enough to prove Lyapunov instability, as is seen by studying the equation

$$
W(q(T), p(T))=W\left(q_{0}, p_{0}\right)+\int_{0}^{T} \dot{W}(q(t), p(t)) d t
$$

and choosing $\left(q_{0}, p_{0}\right)$ in the sector where $W$ is negative, but arbitrarily close to the origin.

Step 2 Employ an argument of Hahn [1967] to show spectral instability.

The sketch of the proof of step 2 is as follows. Since $\epsilon$ is small and the original system is Hamiltonian, the only nontrivial possibility to exclude is the case in which the unperturbed eigenvalues lie on the imaginary axis at nonzero values and that, after perturbation, they remain on the imaginary axis. Indeed, they cannot all move left by Step 1 and $L_{\epsilon}$ cannot have zero eigenvalues since $L_{\epsilon} z=0$ implies $\dot{W}(z, z)=0$. However, in this case, Hahn [1967] shows the existence of at least one periodic orbit, which cannot exist in view of $(6)$ and the fact that $\dot{W}$ is negative definite. The details of these two steps are carried out in $\S \mathbf{3}$ and $\S \mathbf{4}$.

This theorem generalizes in two significant ways. First, it is valid for infinite dimensional systems, where $M, S, R$ and $\Lambda$ are replaced by linear operators. One of course needs some technical conditions to ensure that $W$ has the requisite properties and that the evolution equations generate a semi-group on an appropriate Banach space. For Step 2 one requires, for example, that the spectrum at $\epsilon=0$ be discrete with all eigenvalues having finite multiplicity. To apply this to nonlinear systems under linearization, one also needs to know that the nonlinear system satisfies some "principle of linearized stability"; for example, it has a good invariant manifold theory associated with it.

The second generalization is to systems in block diagonal form but with a nonabelian group. The system (4) is the form that block diagonalization gives with an abelian symmetry group. For a non-abelian group, one gets, roughly speaking, a system consisting of (4) coupled with a Lie-Poisson (generalized rigid body) system. The main step needed in this case is a significant generalization of the Chetaev function. This is carried out in $\S \mathbf{3}$. 
A nonabelian example (with the group $S O(3)$ ) that we consider in $\S \mathbf{6}$ is the rigid body with internal momentum wheels.

The formulation of Theorem $\mathbf{1 . 1}$ and its generalizations is attractive because of the interesting conclusions that can be obtained essentially from energetics alone. If one is willing to make additional assumptions, then there is a formula giving the amount by which simple eigenvalues move off the imaginary axis. One version of this formula, due to MacKay [1991], states that ${ }^{1}$

$$
\operatorname{Re} \lambda_{\epsilon}=\frac{\bar{\xi}(\mathbb{J} B)_{\mathrm{anti}} \xi}{\bar{\xi}^{T} \rrbracket \xi} \epsilon+O\left(\epsilon^{2}\right)
$$

where we write the linearized equations in the form

$$
\dot{z}=L_{\epsilon} z=(\mathbb{J} \mathcal{Q}+\epsilon B) z .
$$

Here, $\lambda_{\epsilon}$ is the perturbed eigenvalue associated with a simple eigenvalue $\lambda_{0}=i \omega_{0}$ on the imaginary axis at $\epsilon=0, \xi$ is a (complex) eigenvector for $L_{0}$ with eigenvalue $\lambda_{0}$, and $(\mathbb{J} B)_{\text {anti }}$ is the antisymmetric part of $\mathbb{J} B$.

In fact, the ratio of quadratic functions in (7) can be replaced by a ratio involving energy-like functions and their time derivatives including the energy itself or the Chetaev function. To actually work out (7) for examples like (1) can involve considerable calculation. See $\S \mathbf{5}$ for details.

What follows is a simple example in which one can carry out the analysis to a large extent directly. We hasten to add that problems like the double spherical pendulum are considerably more complex algebraically and a direct analysis of the eigenvalue movement would not be so simple.

Consider the following gyroscopic system (cf. Chetaev [1961] and Baillieul and Levi [1991])

$$
\left.\begin{array}{l}
\ddot{x}-g \dot{y}+\gamma \dot{x}+\alpha x=0 \\
\ddot{y}+g \dot{x}+\delta \dot{y}+\beta y=0
\end{array}\right\}
$$

which is a special case of (4). Assume $\gamma \geq 0$ and $\delta \geq 0$. For $\gamma=\delta=0$ this system is Hamiltonian with symplectic form

$$
\Omega=d x \wedge d \dot{x}+d y \wedge d \dot{y}-g d x \wedge d y
$$

and the bracket (1.3) where $S=\left[\begin{array}{cc}0 & -g \\ g & 0\end{array}\right]$ and Hamiltonian

$$
H=\frac{1}{2}\left(\dot{x}^{2}+\dot{y}^{2}\right)+\frac{1}{2}\left(\alpha x^{2}+\beta y^{2}\right) .
$$

\footnotetext{
${ }^{1}$ As Mark Levi has pointed out to us, formulae like (1.7) go back to Krein [1950] and Krein also obtained such formulae for periodic orbits (see Levi [1977], formula (18), p. 33).
} 
Note that for $\alpha=\beta$, angular momentum is conserved corresponding to the $S^{1}$ symmetry of $H$. The characteristic polynomial is computed to be

$$
p(\lambda)=\lambda^{4}+(\gamma+\delta) \lambda^{3}+\left(g^{2}+\alpha+\beta+\gamma \delta\right) \lambda^{2}+(\gamma \beta+\delta \alpha) \lambda+\alpha \beta .
$$

Let the characteristic polynomial for the undamped system be denoted $p_{0}$ :

$$
p_{0}(\lambda)=\lambda^{4}+\left(g^{2}+\alpha+\beta\right) \lambda^{2}+\alpha \beta .
$$

Since $p_{0}$ is quadratic in $\lambda^{2}$, its roots are easily found. One gets:

i If $\alpha>0, \beta>0$, then $H$ is positive definite and the eigenvalues are on the imaginary axis; they are coincident in a $1: 1$ resonance for $\alpha=\beta$.

ii If $\alpha$ and $\beta$ have opposite signs, then $H$ has index 1 and there is one eigenvalue pair on the real axis and one pair on the imaginary axis.

iii If $\alpha<0$ and $\beta<0$ then $H$ has index 2. Here the eigenvalues may or may not be on the imaginary axis.

To determine what happens in the last case, let

$$
D=\left(g^{2}+\alpha+\beta\right)^{2}-4 \alpha \beta=g^{4}+2 g^{2}(\alpha+\beta)+(\alpha-\beta)^{2}
$$

be the discriminant, so that the roots of (13) are given by

$$
\lambda^{2}=\frac{1}{2}\left[-\left(g^{2}+\alpha+\beta\right) \pm \sqrt{D}\right]
$$

Thus we arrive at the following conclusions:

a If $D<0$, then there are two roots in the right half plane and two in the left.

b If $D=0$ and $g^{2}+\alpha+\beta>0$, there are coincident roots on the imaginary axis, and if $g^{2}+\alpha+\beta<0$, there are coincident roots on the real axis.

c If $D>0$ and $g^{2}+\alpha+\beta>0$, the roots are on the imaginary axis and if $g^{2}+\alpha+\beta<0$, they are on the real axis.

Thus the case in which $D \geq 0$ and $g^{2}+\alpha+\beta>0$ (i.e., if $g^{2}+\alpha+\beta \geq 2 \sqrt{\alpha \beta}$ ), is one to which the dissipation induced instabilities theorem (Theorem 1.1) applies.

Note that for $g^{2}+\alpha+\beta>0$, if $D$ decreases through zero, a Hamiltonian Hopf bifurcation occurs. For example, as $g$ increases and the eigenvalues move onto the imaginary axis, one speaks of the process as gyroscopic stabilization.

Now we add damping and get

Proposition 1.2 If $\alpha<0, \beta<0, D>0, g^{2}+\alpha+\beta>0$ and least one of $\gamma, \delta$ is strictly positive, then for (9), there is exactly one pair of eigenvalues in the strict right half plane. 
Proof We use the Routh-Hurwitz criterion (see Gantmacher [1959, vol. 2]), which states that the number of strict right half plane roots of the polynomial

$$
\lambda^{4}+\rho_{1} \lambda^{3}+\rho_{2} \lambda^{2}+\rho_{3} \lambda+\rho_{4}
$$

equals the number of sign changes in the sequence

$$
\left\{1, \rho_{1}, \frac{\rho_{1} \rho_{2}-\rho_{3}}{\rho_{1}}, \frac{\rho_{3}\left(\rho_{1} \rho_{2}-\rho_{3}\right)-\rho_{1} \rho_{4}}{\rho_{1} \rho_{2}-\rho_{3}}, \rho_{4}\right\} \text {. }
$$

For our case, $\rho_{1}=\gamma+\delta>0, \rho_{2}=g^{2}+\alpha+\beta+\gamma \delta>0, \rho_{3}=\gamma \beta+\alpha \delta<0$ and $\rho_{4}=\alpha \beta>0$, so the sign sequence (14) is

$$
\{+,+,+,-,+\} \text {. }
$$

Thus, there are two roots in the right half plane.

This proof confirms the result of Theorem 1.1. It gives more information, but for complex systems, this method, while instructive, may be difficult or impossible to implement, while the method of Theorem $\mathbf{1 . 1}$ is easy to implement. One can also use methods of Krein and MacKay to get the result of the above proposition and get, in fact, additional information about how far the eigenvalues move to the right as a function of the size of the dissipation. We shall present this technique in $\S \mathbf{5}$. Again, this technique gives more specific information, but is harder to implement, as it requires more hypotheses (simplicity of eigenvalues) and requires one to compute the corresponding eigenvector of the unperturbed system, which may not be a simple task.

Example An instructive special case of the system (1.9) is the system of equations describing a bead in equilibrium at the center of a rotating circular plate driven with angular velocity $\omega$ and subject to a central restoring force. (These equations may also be regarded as the linearized equations of motion for a rotating spherical pendulum in a gravitational field - see Baillieul and Levi [1991].) Let $x$ and $y$ denote the position of the bead in a rotating coordinate system fixed in the plate. The Lagrangian is then

$$
\frac{1}{2}\left(x_{t}-\omega y\right)^{2}+\left(y_{t}+\omega x\right)^{2}-\frac{1}{2} k\left(x^{2}+y^{2}\right)
$$

and the equations of motion without damping are

$$
\begin{aligned}
& x_{t t}-2 \omega y_{t}+\left(k-\omega^{2}\right) x=0 \\
& y_{t t}+2 \omega x_{t}+\left(k-\omega^{2}\right) x=0 .
\end{aligned}
$$

Thus for $\omega^{2}>k$ the system is gyroscopically stable and the addition of Rayleigh damping induces spectral instability.

It is interesting to speculate on the effect of damping on the Hamiltonian Hopf bifurcation in view of these general results and in particular, this example. 
For instance, suppose $g^{2}+\alpha+\beta>0$ and we allow $D$ to increase so a Hamiltonian Hopf bifurcation occurs in the undamped system. Then the above sign sequence does not change, so no bifurcation occurs in the damped system; the system is unstable and the Hamiltonian Hopf bifurcation just enhances this instability. However, if we simulate forcing or control by allowing one of $\gamma$ or $\delta$ to be negative, but still small, then the sign sequence is more complex and one can get, for example, the Hamiltonian Hopf bifurcation breaking up into two nearly coincident Hopf bifurcations. These remarks are consistent with van Gils, Krupa, and Langford [1990].

The preceding discussion assumes that the equilibrium of the original nonlinear equation being linearized is independent of $\epsilon$. In general of course this is not true, but it can be dealt with as follows. Consider the nonlinear equation

$$
\dot{x}=f(x, \epsilon)
$$

on a Banach space, say. Assume $f(0,0)=0$ and $x(\epsilon)$ is a curve of equilibria with $x(0)=0$. By implicitly differentiating $f(x(\epsilon), \epsilon)=0$ we find that the linearized equations at $x(\epsilon)$ are given by

$$
\begin{aligned}
(\dot{\delta x)}= & \mathbf{D}_{x} f(x(\epsilon), \epsilon) \delta x \\
= & \mathbf{D}_{x} f(0,0) \delta x+\epsilon\left[\mathbf{D}_{x \epsilon}^{2} f(0,0) \delta x+\mathbf{D}_{x}^{2} f(0,0)\left(x^{\prime}(0), \delta x\right)\right] \\
& +O\left(\epsilon^{2}\right)
\end{aligned}
$$

where $x^{\prime}(0)=-\mathbf{D}_{x} f(0,0)^{-1} f_{\epsilon}(0,0)$, assuming that $\mathbf{D}_{x} f(0,0)$ is invertible; i.e., we are not at a bifurcation point. In principle then, (1.17) is computable in terms of data at $(0,0)$ and our general theory applies.

A situation of interest for KAM theory is the study of the dynamics near an elliptic fixed point of a Hamiltonian system with several degrees of freedom. The usual hypothesis is that the equations linearized about this fixed point have a spectrum that lies on the imaginary axis and that the second variation of the Hamiltonian at this fixed point is indefinite. Our result says that these elliptic fixed points become spectrally unstable with the addition of (small) damping. It would be of interest to investigate the role of our result, and associated system symmetry breaking results (see, for example, Guckenheimer and Mahalov [1992]), for these systems and in the context of Hamiltonian normal forms, more thoroughly (see, for example, Haller [1992]). In particular, the relation between the results here and the phenomenon of capture into resonance would be of considerable interest.

There are a number of other topics that should be investigated in the future. For example, the present results would be interesting to apply to some fluid systems. The cases of interest here, in which eigenvalues lie on the imaginary axis, but the second variation of the relevant energy quantity is indefinite, occur for circular rotating liquid drops (Lewis, Marsden, and Ratiu [1987] and Lewis [1989]), for shear flow in a stratified fluid with Richardson number between 1/4 and 1 (Abarbanel et al. [1986]), in plasma dynamics (Morrison and Kotschenreuther [1989], Kandrup [1991], and Kandrup and Morrison [1992]), and for rotating strings. In each of these examples, there are essential pde difficulties that need to be overcome, and we have written the present paper to adapt to that situation as far as possible. One infinite 
dimensional example that we consider is the case of a rotating rod in $\S \mathbf{6}$, but it can be treated by essentially finite dimensional methods, and the pde difficulties we were alluding to do not occur. We also point out that some of the same effects as seen here are also found in reversible (but non-Hamiltonian) systems; see O'Reilly et. al. [1993].

Acknowledgments We thank Stuart Antman, John Baillieul, György Haller, Mark Levi, Debbie Lewis, John Maddocks, Lisheng Wang, and Steve Wiggins for helpful comments. In particular, we thank Debbie Lewis for helpful comments on the linearized equations in the block diagonalization theory. We also thank the Fields Institute for providing the opportunity to meet in pleasant surroundings.

\section{The Energy-Momentum Method}

Our framework for the energy-momentum method will be that of simple mechanical systems with symmetry. We choose as the phase space $P=T Q$ or $P=T^{*} Q$, a tangent or cotangent bundle of a configuration space $Q$. Assume there is a Riemannian metric $\langle\langle\rangle$,$\rangle on Q$, that a Lie group $G$ acts on $Q$ by isometries (and so $G$ acts symplectically on $T Q$ by tangent lifts and on $T^{*} Q$ by cotangent lifts). The Lagrangian is taken to be of the form

$$
L(q, v)=\frac{1}{2}\|v\|_{q}^{2}-V(q)
$$

or equivalently, the Hamiltonian is

$$
H(q, p)=\frac{1}{2}\|p\|_{q}^{2}+V(q)
$$

where $\|\cdot\|_{q}$ is the norm on $T_{q} Q$ or the one induced on $T_{q}^{*} Q$, and where $V$ is a $G$-invariant potential.

With a slight abuse of notation, we write either $(q, v)$ or $v_{q}$ for a vector based at $q \in Q$ and $z=(q, p)$ or $z=p_{q}$ for a covector based at $q \in Q$. The pairing between $T_{q}^{*} Q$ and $T_{q} Q$ is written

$$
\left\langle p_{q}, v_{q}\right\rangle, \quad\langle p, v\rangle \quad \text { or } \quad\langle(q, p),(q, v)\rangle .
$$

Other natural pairings between spaces and their duals are also denoted $\langle$,$\rangle .$

The standard momentum map for simple mechanical $G$-systems is

$$
\begin{aligned}
\mathbf{J}: T Q \rightarrow \mathfrak{g}^{*}, \quad \text { where } \quad\langle\mathbf{J}(q, v), \xi\rangle=\left\langle\left\langle v, \xi_{Q}(q)\right\rangle\right\rangle \\
\text { or } \quad \mathbf{J}: T^{*} Q \rightarrow \mathfrak{g}^{*}, \quad \text { where } \quad\langle\mathbf{J}(q, p), \xi\rangle=\left\langle p, \xi_{Q}(q)\right\rangle
\end{aligned}
$$

where $\xi_{Q}$ denotes the infinitesimal generator of $\xi \in \mathfrak{g}$ on $Q$. We use the same notation for $\mathbf{J}$ regarded as a map on either the cotangent or the tangent space; which is meant will be clear from the context. For future use, we set $\mathfrak{g} \cdot q=\left\{\xi_{Q}(q) \mid \xi \in \mathfrak{g}\right\} \subset T_{q} Q$. 
Assume that $G$ acts freely on $Q$ so we can regard $Q \rightarrow Q / G$ as a principal $G$ bundle. A refinement shows that one really only needs the action of $G_{\mu}$ on $Q$ to be free and all the constructions can be done in terms of the bundle $Q \rightarrow Q / G_{\mu}$; here, $G_{\mu}$ is the isotropy subgroup for $\mu \in \mathfrak{g}^{*}$ for the coadjoint action of $G$ on $\mathfrak{g}^{*}$. Recall that for abelian groups, $G=G_{\mu}$. However, we do the constructions for the action of the full group $G$ for simplicity of exposition.

For each $q \in Q$, let the locked inertia tensor be the map $\mathbb{I}(q): \mathfrak{g} \rightarrow \mathfrak{g}^{*}$ defined by

$$
\langle\mathbb{I}(q) \eta, \zeta\rangle=\left\langle\left\langle\eta_{Q}(q), \zeta_{Q}(q)\right\rangle\right\rangle .
$$

Since the action is free, $\mathbb{I}(q)$ is indeed an inner product. The terminology comes from the fact that for coupled rigid or elastic systems, $\mathbb{I}(q)$ is the classical moment of inertia tensor of the corresponding rigid system. Most of the results of this paper hold in the infinite as well as the finite dimensional case. To expedite the exposition, we give many of the formulae in coordinates for the finite dimensional case. For instance,

$$
\mathbb{I}_{a b}=g_{i j} A_{a}^{i} A_{b}^{j}
$$

where we write

$$
\left[\xi_{Q}(q)\right]^{i}=A_{a}^{i}(q) \xi^{a}
$$

relative to coordinates $q^{i}, i=1,2, \ldots, n$ on $Q$ and a basis $e_{a}, a=1,2, \ldots, m$ of $\mathfrak{g}$.

Define the map $\alpha: T Q \rightarrow \mathfrak{g}$ which assigns to each $(q, v)$ the corresponding angular velocity of the locked system:

$$
\alpha(q, v)=\mathbb{I}(q)^{-1}(\mathbf{J}(q, v)) .
$$

In coordinates,

$$
\alpha^{a}=\mathbb{I}^{a b} g_{i j} A_{b}^{i} v^{j} .
$$

The map (26) is a connection on the principal $G$-bundle $Q \rightarrow Q / G$. In other words, $\alpha$ is $G$-equivariant and satisfies $\alpha\left(\xi_{Q}(q)\right)=\xi$, both of which are readily verified. In checking equivariance one uses invariance of the metric, equivariance of $\mathbf{J}: T Q \rightarrow \mathfrak{g}^{*}$, and equivariance of $\mathbb{I}$ in the sense of a map $\mathbb{I}: Q \rightarrow \mathcal{L}\left(\mathfrak{g}, \mathfrak{g}^{*}\right)$ (i.e., the space of linear maps of $\mathfrak{g}$ to $\left.\mathfrak{g}^{*}\right)$, namely $\mathbb{I}(g \cdot q) \cdot A d_{g} \xi=A d_{g^{-1}}^{*} \mathbb{I}(q) \cdot \xi$.

We call $\alpha$ the mechanical connection, as in Simo, Lewis and Marsden [1991]. The horizontal space of the connection $\alpha$ is given by

$$
\operatorname{hor}_{q}=\{(q, v) \mid \mathbf{J}(q, v)=0\}
$$

i.e., the space orthogonal to the $G$-orbits. The vertical space consists of vectors that are mapped to zero under the projection $Q \rightarrow S=Q / G$; i.e.,

$$
\operatorname{ver}_{q}=\left\{\xi_{Q}(q) \mid \xi \in \mathfrak{g}\right\}=\mathfrak{g} \cdot q \text {. }
$$


For each $\mu \in \mathfrak{g}^{*}$, define the 1 -form $\alpha_{\mu}$ on $Q$ by

$$
\left\langle\alpha_{\mu}(q), v\right\rangle=\langle\mu, \alpha(q, v)\rangle
$$

i.e.,

$$
\left(\alpha_{\mu}\right)_{i}=g_{i j} A_{b}^{j} \mu_{a} \mathbb{I}^{a b}
$$

One sees from $\alpha\left(\xi_{Q}(q)\right)=\xi$ that $\alpha_{\mu}$ takes values in $\mathbf{J}^{-1}(\mu)$. The horizontal-vertical decomposition of a vector $(q, v) \in T_{q} Q$ is given by

$$
v=\operatorname{hor}_{q} v+\operatorname{ver}_{q} v
$$

where

$$
\operatorname{ver}_{q} v=[\alpha(q, v)]_{Q}(q) \text { and } \operatorname{hor}_{q} v=v-\operatorname{ver}_{q} v .
$$

Notice that hor $: T Q \rightarrow \mathbf{J}^{-1}(0)$ and as such, it may be regarded as a velocity shift.

The amended potential $V_{\mu}$ is defined by

$$
V_{\mu}(q)=V(q)+\frac{1}{2}\left\langle\mu, \mathbb{I}(q)^{-1} \mu\right\rangle
$$

In coordinates,

$$
V_{\mu}(q)=V(q)+\frac{1}{2} \mathbb{I}^{a b}(q) \mu_{a} \mu_{b}
$$

We recall from Abraham and Marsden [1978] or Simo, Lewis, and Marsden [1991] that in a symplectic manifold $(P, \Omega)$, a point $z_{e} \in P$ is called a relative equilibrium if

$$
X_{H}\left(z_{e}\right) \in T_{z_{e}}\left(G \cdot z_{e}\right)
$$

i.e., if the Hamiltonian vector field at $z_{e}$ points in the direction of the group orbit through $z_{e}$. The Relative Equilibrium Theorem states that if $z_{e} \in P$ and $z_{e}(t)$ is the dynamic orbit of $X_{H}$ with $z_{e}(0)=z_{e}$ and $\mu=\mathbf{J}\left(z_{e}\right)$, then the following conditions are equivalent

1. $z_{e}$ is a relative equilibrium

2. $z_{e}(t) \in G_{\mu} \cdot z_{e} \subset G \cdot z_{e}$

3. there is a $\xi \in \mathfrak{g}$ such that $z_{e}(t)=\exp (t \xi) \cdot z_{e}$

4. there is a $\xi \in \mathfrak{g}$ such that $z_{e}$ is a critical point of the augmented Hamiltonian

$$
H_{\xi}(z):=H(z)-\langle\mathbf{J}(z)-\mu, \xi\rangle
$$

5. $z_{e}$ is a critical point of $H \times \mathbf{J}: P \rightarrow \mathbb{R} \times \mathfrak{g}^{*}$, the energy-momentum map

6. $z_{e}$ is a critical point of $H \mid \mathbf{J}^{-1}(\mu)$ 
7. $z_{e}$ is a critical point of $H \mid \mathbf{J}^{-1}(\mathcal{O})$, where $\mathcal{O}=G \cdot \mu \in \mathfrak{g}^{*}$

8. $\left[z_{e}\right] \in P_{\mu}$ is a critical point of the reduced Hamiltonian $H_{\mu}$.

Straightforward algebraic manipulation shows that $H_{\xi}$ can be rewritten as follows

$$
H_{\xi}(q, v)=K_{\xi}(q, v)+V_{\xi}(q)+\langle\mu, \xi\rangle
$$

where

$$
K_{\xi}(q, v)=\frac{1}{2}\left\|v-\xi_{Q}(q)\right\|^{2}
$$

and where

$$
V_{\xi}(q)=V(q)-\frac{1}{2}\langle\xi, \mathbb{I}(q) \xi\rangle
$$

These identities show the following.

Proposition 2.1 A point $z_{e}=\left(q_{e}, v_{e}\right)$ is a relative equilibrium if and only if there is a $\xi \in \mathfrak{g}$ such that

1. $v_{e}=\xi_{Q}\left(q_{e}\right)$ and

2. $q_{e}$ is a critical point of $V_{\xi}$.

The functions $K_{\xi}$ and $V_{\xi}$ are called the augmented kinetic and potential energies respectively. The main point of this proposition is that it reduces the job of finding relative equilibria to finding critical points of $V_{\xi}$.

Relative equilibria may also be characterized by the amended potential. One has the following identity:

$$
H(q, p)=K_{\mu}(q, p)+V_{\mu}(q)
$$

where

$$
K_{\mu}(q, p)=\frac{1}{2}\left\|p-\alpha_{\mu}(q)\right\|^{2},
$$

for $(q, p) \in \mathbf{J}^{-1}(\mu)$. This leads to the following:

Proposition 2.2 A point $\left(q_{e}, v_{e}\right)$ with $\mathbf{J}\left(q_{e}, v_{e}\right)=\mu$ is a relative equilibrium if and only if

1. $v_{e}=\xi_{Q}\left(q_{e}\right)$ where $\xi=\mathbb{I}^{-1}(q) \mu$ and

2. $q_{e}$ is a critical point of $V_{\mu}$. 
Next, we summarize the energy-momentum method of Simo, Posbergh and Marsden [1990, 1991], Simo, Lewis and Marsden [1991], on the purely Lagrangian side, Lewis [1992], and in a control theoretic context, Wang and Krishnaprasad [1992]. This is a technique for determining the stability of relative equilibria and for putting the equations of motion linearized at a relative equilibrium, into normal form. This normal form is based on a special decomposition into rigid and internal variables.

We confine ourselves to the regular case; that is, we assume $z_{e}$ is a relative equilibrium that is also a regular point (i.e., $\mathfrak{g}_{z_{e}}=\{0\}$, or $z_{e}$ has a discrete isotropy group) and $\mu=\mathbf{J}\left(z_{e}\right)$ is a generic point in $\mathfrak{g}^{*}$ (i.e., its orbit is of maximal dimension). We are seeking conditions for stability of $z_{e}$ modulo $G_{\mu}$.

The energy-momentum method is as follows: Choose a subspace $\mathcal{S} \subset \operatorname{kerDJ}\left(q_{e}, v_{e}\right)$ that is also transverse to the $G_{\mu}$ orbit of $\left(q_{e}, v_{e}\right)$

a find $\xi \in \mathfrak{g}$ such that $\delta H_{\xi}\left(z_{e}\right)=0$

b test $\delta^{2} H_{\xi}\left(z_{e}\right)$ for definiteness on $\mathcal{S}$.

Theorem 2.3 The Energy-Momentum Theorem. If $\delta^{2} H_{\xi}\left(z_{e}\right)$ is definite, then $z_{e}$ is $G_{\mu}$-orbitally stable in $\mathbf{J}^{-1}(\mu)$ and $G$-orbitally stable in $P$.

For simple mechanical systems, one way to choose $\mathcal{S}$ is as follows. Let

$$
\mathcal{V}=\left\{\delta q \in T_{q_{e}} Q \mid\left\langle\left\langle\delta q, \chi_{Q}\left(q_{e}\right)\right\rangle\right\rangle=0 \quad \text { for all } \quad \chi \in \mathfrak{g}_{\mu}\right\},
$$

the metric orthogonal complement of the tangent space to the $G_{\mu}$-orbit in $Q$. Let

$$
\mathcal{S}=\left\{\delta z \in \operatorname{ker} \mathbf{D J}\left(z_{e}\right) \mid T \pi_{Q} \cdot \delta z \in \mathcal{V}\right\}
$$

where $\pi_{Q}: T^{*} Q=P \rightarrow Q$ is the projection.

If the energy-momentum method is applied to mechanical systems with Hamiltonian $H$ of the form kinetic energy $(K)$ plus potential $(V)$, under hypotheses given below, it is possible to choose variables in a way that makes the determination of stability conditions sharper and more computable. In this set of variables (with the conservation of momentum constraint and a gauge symmetry constraint imposed on $\mathcal{S})$, the second variation of $\delta^{2} H_{\xi}$ block diagonalizes; schematically

$$
\delta^{2} H_{\xi}=\left[\begin{array}{c}
{\left[\begin{array}{c}
\text { coadjoint } \\
\text { orbit block }
\end{array}\right]} \\
0
\end{array}\right]
$$

Furthermore, the internal vibrational block takes the form

$$
\left[\begin{array}{c}
\text { Internal vibration } \\
\text { block }
\end{array}\right]=\left[\begin{array}{cc}
\delta^{2} V_{\mu} & 0 \\
0 & \delta^{2} K_{\mu}
\end{array}\right]
$$

where $V_{\mu}$ is the amended potential defined earlier, and $K_{\mu}$ is a momentum shifted kinetic energy. Thus, formal stability is equivalent to $\delta^{2} V_{\mu}>0$ and that the overall structure is stable when viewed as a rigid structure, which, as far as stability is 
concerned, separates out the overall rigid body motions from the internal motions of the system under consideration.

To define the rigid-internal splitting, we begin with a splitting in configuration space. Consider (at a relative equilibrium) the space $\mathcal{V}$ defined above as the metric orthogonal complement to $\mathfrak{g}_{\mu} \cdot q$ in $T_{q} Q$. Here we drop the subscript $e$ for notational convenience. Then we split

$$
\mathcal{V}=\mathcal{V}_{\mathrm{RIG}} \oplus \mathcal{V}_{\mathrm{INT}}
$$

as follows. Define

$$
\mathcal{V}_{\mathrm{RIG}}=\left\{\eta_{Q}(q) \in T_{q} Q \mid \eta \in \mathfrak{g}_{\mu}^{\perp}\right\}
$$

where $\mathfrak{g}_{\mu}^{\perp}$ is the orthogonal complement to $\mathfrak{g}_{\mu}$ in $\mathfrak{g}$ with respect to the locked inertia metric. (This choice of orthogonal complement depends on $q$, but we do not include this in the notation.) From (39) it is clear that $\mathcal{V}_{\text {RIG }} \subset \mathcal{V}$ and that $\mathcal{V}_{\text {RIG }}$ has the dimension of the coadjoint orbit through $\mu$. Next, define

$$
\mathcal{V}_{\mathrm{INT}}=\left\{\delta q \in \mathcal{V} \mid\langle\eta,[\mathbf{D} \mathbb{I}(q) \cdot \delta q] \cdot \xi\rangle=0 \quad \text { for all } \quad \eta \in \mathfrak{g}_{\mu}^{\perp}\right\}
$$

where $\xi=\mathbb{I}(q)^{-1} \mu$. An equivalent definition is $\mathcal{V}_{\mathrm{INT}}=\left\{\delta q \in \mathcal{V} \mid\left[\mathbf{D} \mathbb{I}(q)^{-1} \cdot \delta q\right] \cdot \mu \in\right.$ $\left.\mathfrak{g}_{\mu}\right\}$. The definition of $\mathcal{V}_{\text {INT }}$ has an interesting mechanical interpretation in terms of the objectivity of the centrifugal force in case $G=S O(3)$; see Simo, Lewis and Marsden [1991].

Define the Arnold form $\mathcal{A}_{\mu}: \mathfrak{g}_{\mu}^{\perp} \times \mathfrak{g}_{\mu}^{\perp} \rightarrow \mathbb{R}$ by

$$
\mathcal{A}_{\mu}(\eta, \zeta)=\left\langle a d_{\eta}^{*} \mu, \chi_{(q, \mu)}(\zeta)\right\rangle=\left\langle\mu, a d_{\eta} \chi_{(q, \mu)}(\zeta)\right\rangle,
$$

where $\chi_{(q, \mu)}: \mathfrak{g}_{\mu}^{\perp} \rightarrow \mathfrak{g}$ is defined by $\chi_{(q, \mu)}(\zeta)=\mathbb{I}(q)^{-1} a d_{\zeta}^{*} \mu+a d_{\zeta} \mathbb{I}(q)^{-1} \mu$. The Arnold form appears in Arnold's [1966] stability analysis of relative equilibria in the special case $Q=G$. At a relative equilibrium, the form $\mathcal{A}_{\mu}$ is symmetric, as is verified either directly or by recognizing it as the second variation of $V_{\mu}$ on $\mathcal{V}_{\text {RIG }} \times \mathcal{V}_{\text {RIG }}$.

At a relative equilibrium, the form $\mathcal{A}_{\mu}$ is degenerate as a symmetric bilinear form on $\mathfrak{g}_{\mu}^{\perp}$ when there is a non-zero $\zeta \in \mathfrak{g}_{\mu}^{\perp}$ such that

$$
\mathbb{I}(q)^{-1} a d_{\zeta}^{*} \mu+a d_{\zeta} \mathbb{I}(q)^{-1} \mu \in \mathfrak{g}_{\mu} ;
$$

in other words, when $\mathbb{I}(q)^{-1}: \mathfrak{g}^{*} \rightarrow \mathfrak{g}$ has a nontrivial symmetry relative to the (coadjoint, adjoint) action of $\mathfrak{g}$ (restricted to $\mathfrak{g}_{\mu}^{\perp}$ ) on the space of linear maps from $\mathfrak{g}^{*}$ to $\mathfrak{g}$. (When one is not at a relative equilibrium, we say the Arnold form is non-degenerate when $\mathcal{A}_{\mu}(\eta, \zeta)=0$ for all $\eta \in \mathfrak{g}_{\mu}^{\perp}$ implies $\zeta=0$.) This means, for $G=S O(3)$ that $\mathcal{A}_{\mu}$ is non-degenerate if $\mu$ is not in a multidimensional eigenspace of $\mathbb{I}^{-1}$. Thus, if the locked body is not symmetric (i.e., a Lagrange top), then the Arnold form is non-degenerate.

Proposition 2.4 If the Arnold form is non-degenerate, then

$$
\mathcal{V}=\mathcal{V}_{\mathrm{RIG}} \oplus \mathcal{V}_{\mathrm{INT}}
$$


Indeed, non-degeneracy of the Arnold form implies $\mathcal{V}_{\text {RIG }} \cap \mathcal{V}_{\text {INT }}=\{0\}$ and, at least in the finite dimensional case, a dimension count gives (43). In the infinite dimensional case, the relevant ellipticity conditions are needed.

The split (43) can now be used to induce a split of the phase space

$$
\mathcal{S}=\mathcal{S}_{\mathrm{RIG}} \oplus \mathcal{S}_{\mathrm{INT}}
$$

Using a more mechanical viewpoint, Simo, Lewis and Marsden [1991] show how $\mathcal{S}_{\mathrm{RIG}}$ can be defined by extending $\mathcal{V}_{\mathrm{RIG}}$ from positions to momenta using superposed rigid motions. For our purposes, the important characterization of $\mathcal{S}_{\mathrm{RIG}}$ is via the mechanical connection:

$$
\mathcal{S}_{\mathrm{RIG}}=T_{q} \alpha_{\mu} \cdot \mathcal{V}_{\mathrm{RIG}}
$$

so $\mathcal{S}_{\mathrm{RIG}}$ is isomorphic to $\mathcal{V}_{\mathrm{RIG}}$. Since $\alpha_{\mu}$ maps $Q$ to $\mathbf{J}^{-1}(\mu)$ and $\mathcal{V}_{\mathrm{RIG}} \subset \mathcal{V}$, we get $\mathcal{S}_{\text {RIG }} \subset \mathcal{S}$. Define

$$
\mathcal{S}_{\mathrm{INT}}=\left\{\delta z \in \mathcal{S} \mid \delta q \in \mathcal{V}_{\mathrm{INT}}\right\}
$$

then (44) holds if the Arnold form is non-degenerate. Next, we write

$$
\mathcal{S}_{\mathrm{INT}}=\mathcal{W}_{\mathrm{INT}} \oplus \mathcal{W}_{\mathrm{INT}}^{\dagger}
$$

where $\mathcal{W}_{\text {INT }}$ and $\mathcal{W}_{\text {INT }}^{\dagger}$ are defined as follows:

$$
\mathcal{W}_{\mathrm{INT}}=T_{q} \alpha_{\mu} \cdot \mathcal{V}_{\mathrm{INT}} \quad \text { and } \quad \mathcal{W}_{\mathrm{INT}}^{\dagger}=\left\{\operatorname{ver}(\gamma) \mid \gamma \in[\mathfrak{g} \cdot q]^{0}\right\}
$$

where $\mathfrak{g} \cdot q=\left\{\zeta_{Q}(q) \mid \zeta \in \mathfrak{g}\right\}, \quad[\mathfrak{g} \cdot q]^{0} \subset T_{q}^{*} Q$ is its annihilator, and $\operatorname{ver}(\gamma) \in T_{z}\left(T^{*} Q\right)$ is the vertical lift of $\gamma \in T_{q}^{*} Q$; in coordinates, $\operatorname{ver}\left(q^{i}, \gamma_{j}\right)=\left(q^{i}, p_{j}, 0, \gamma_{j}\right)$. The vertical lift is given intrinsically by taking the tangent to the curve $\sigma(s)=z+s \gamma$ at $s=0$.

Theorem 2.5 Block Diagonalization Theorem Assume that the Arnold form is nondegenerate. Then in the splittings introduced above at a relative equilibrium, $\delta^{2} H_{\xi}\left(z_{e}\right)$ and the symplectic form $\Omega_{z_{e}}$ have the following form:

$$
\delta^{2} H_{\xi}\left(z_{e}\right)=\left[\begin{array}{ccc}
{\left[\begin{array}{c}
\text { Arnold } \\
\text { form }
\end{array}\right]} & 0 & 0 \\
0 & \delta^{2} V_{\mu} & 0 \\
0 & 0 & \delta^{2} K_{\mu}
\end{array}\right]
$$

and

$$
\Omega_{z_{e}}=\left[\begin{array}{ccc}
{\left[\begin{array}{c}
\text { coadjoint orbit } \\
\text { symplectic form }
\end{array}\right]} & {\left[\begin{array}{c}
\text { internal rigid } \\
\text { coupling }
\end{array}\right]} & 0 \\
-\left[\begin{array}{c}
\text { internal rigid } \\
\text { coupling }
\end{array}\right] & S & \mathcal{I} \\
0 & -\mathcal{I}^{T} & 0
\end{array}\right]
$$


where the columns represent elements of $\mathcal{S}_{\mathrm{RIG}}, \mathcal{W}_{\mathrm{INT}}$ and $\mathcal{W}_{\mathrm{INT}}^{\dagger}$, respectively, and $\mathcal{I}: \mathcal{W}_{\text {INT }}^{\dagger} \rightarrow \mathcal{W}_{\text {INT }}^{*}$ is the isomorphism given as follows: Let vert $(\gamma) \in \mathcal{W}_{\text {INT }}^{\dagger}$ where $\gamma \in[\mathfrak{g} \cdot q]^{0}$ and let $\delta q \in \mathcal{V}_{\mathrm{INT}}$; then

$$
\left\langle\mathcal{I}(\operatorname{vert}(\gamma)), T_{q} \alpha_{\mu} \cdot \delta q\right\rangle=\langle\gamma, \delta q\rangle
$$

As far as stability is concerned, we have the following consequence of block diagonalization.

Theorem 2.6 Reduced Energy-Momentum Method Let $z_{e}=\left(q_{e}, p_{e}\right)$ be a (cotangent) relative equilibrium and assume that the internal variables are not trivial; i.e., $\mathcal{V}_{\mathrm{INT}} \neq\{0\}$. If $\delta^{2} H_{\xi}\left(z_{e}\right)$ is definite, then it must be positive definite. Necessary and sufficient conditions for $\delta^{2} H_{\xi}\left(z_{e}\right)$ to be positive definite are

1. the Arnold form is positive definite on $\mathcal{V}_{\mathrm{RIG}}$ and

2. $\delta^{2} V_{\mu}\left(q_{e}\right)$ is positive definite on $\mathcal{V}_{\mathrm{INT}}$.

This follows since $\delta^{2} K_{\mu}$ is positive definite and $\delta^{2} H_{\xi}$ has the above block diagonal structure.

In examples, it is this form of the energy-momentum method that is normally easiest to use.

A straightforward calculation establishes the useful relation

$$
\delta^{2} V_{\mu}\left(q_{e}\right) \cdot(\delta q, \delta q)=\delta^{2} V_{\xi}\left(q_{e}\right) \cdot(\delta q, \delta q)+\left\langle\left(\mathbf{D} \mathbb{I}\left(q_{e}\right) \cdot \delta q\right) \xi,\left(\mathbb{I}\left(q_{e}\right)^{-1} \circ \mathbf{D} \mathbb{I}\left(q_{e}\right) \cdot \delta q\right) \xi\right\rangle
$$

and the correction term is positive. Thus, if $\delta^{2} V_{\xi}\left(q_{e}\right)$ is positive definite, then so is $\delta^{2} V_{\mu}\left(q_{e}\right)$, but not necessarily conversely. Thus, $\delta^{2} V_{\mu}\left(q_{e}\right)$ gives sharp conditions for stability (in the sense of Theorem $\mathbf{2 . 3}$ ), while $\delta^{2} V_{\xi}$ gives only sufficient conditions.

Using the notation $\zeta=\left[\mathbf{D} \mathbb{I}^{-1}\left(q_{e}\right) \cdot \delta q\right] \mu \in \mathfrak{g}_{\mu}$ (see the comments following (41)), observe that the "correcting term" in (49) is given by $\left\langle\mathbb{I}\left(q_{e}\right) \zeta, \zeta\right\rangle=\left\langle\left\langle\zeta_{Q}\left(q_{e}\right), \zeta_{Q}\left(q_{e}\right)\right\rangle\right\rangle$.

One of the most interesting aspects of block diagonalization is that the rigidinternal splitting also brings the symplectic structure into normal form. We already gave the general structure of this and here we provide a few more details. We emphasise once more that this implies that the equations of motion are also put into normal form and this is useful for studying eigenvalue movement for purposes of bifurcation theory. For example, for abelian groups, the linearized equations of motion take the gyroscopic form:

$$
M \ddot{q}+S \dot{q}+\Lambda q=0
$$

where $M$ is a positive definite symmetric matrix (the mass matrix), $\Lambda$ is symmetric (the potential term) and $S$ is skew (the gyroscopic, or magnetic term). This second order form is particularly useful for finding eigenvalues of the linearized equations (see, for example, Bloch, Krishnaprasad, Marsden and Ratiu [1991]).

To make the normal form of the symplectic structure explicit, we need some preliminary results. See Simo, Lewis and Marsden [1991] for the proofs. 
Lemma 2.7 Let $\Delta q=\eta_{Q}\left(q_{e}\right) \in \mathcal{V}_{\mathrm{RIG}}$ and $\Delta z=T \alpha_{\mu} \cdot \Delta q \in \mathcal{S}_{\mathrm{RIG}}$. Then

$$
\Delta z=\operatorname{vert}\left[\mathbb{F} L\left(\zeta_{Q}\left(q_{e}\right)\right)\right]-T^{*} \eta_{Q}\left(q_{e}\right) \cdot p_{e}
$$

where $\zeta=\mathbb{I}\left(q_{e}\right)^{-1} a d_{\eta}^{*} \mu$, vert denotes the vertical lift, and $\mathbb{F} L$ denotes the fiber derivative.

Lemma 2.8 For any $\delta z \in T_{z_{e}} P$,

$$
\Omega\left(z_{e}\right)(\Delta z, \delta z)=\left\langle\left[\mathbf{D J}\left(z_{e}\right) \cdot \delta z\right], \eta\right\rangle-\left\langle\left\langle\zeta_{Q}\left(q_{e}\right), \delta q\right\rangle\right\rangle
$$

If $\delta z \in \mathcal{S}_{\mathrm{INT}}$, then it lies in ker DJ, so we get the internal-rigid interaction terms:

$$
\Omega\left(z_{e}\right)(\Delta z, \delta z)=-\left\langle\left\langle\zeta_{Q}\left(q_{e}\right), \delta q\right\rangle\right\rangle=-\left\langle\mu_{e},[\eta, \alpha(\delta q)]\right\rangle .
$$

Since these involve only $\delta q$ and not $\delta p$, there is a zero in the last slot in the first row of $\Omega$ and so we can define the operator $C$ by $(2.34):\langle C(\delta q), \Delta q\rangle:=\Omega\left(z_{e}\right)(\Delta z, \delta z)$.

Lemma 2.9 The rigid-rigid terms in $\Omega$ are

$$
\Omega\left(z_{e}\right)\left(\Delta_{1} z, \Delta_{2} z\right)=-\left\langle\mu,\left[\eta_{1}, \eta_{2}\right]\right\rangle,
$$

which is the coadjoint orbit symplectic structure.

Next, we turn to the magnetic terms:

Lemma 2.10 Let $\delta_{1} z=T \alpha_{\mu} \cdot \delta_{1} q$ and $\delta_{2} z=T \alpha_{\mu} \cdot \delta_{2} q \in \mathcal{W}_{\text {INT }}$, where $\delta_{1} q, \delta_{2} q \in$ $\mathcal{V}_{\mathrm{INT}}$. Then

$$
\Omega\left(z_{e}\right)\left(\delta_{1} z, \delta_{2} z\right)=-\mathbf{d} \alpha_{\mu}\left(\delta_{1} q, \delta_{2} q\right)
$$

If we define the one form $\alpha_{\xi}$ by $\alpha_{\xi}(q)=\mathbb{F} L\left(\xi_{Q}(q)\right)$, then the definition of $\mathcal{V}_{\text {INT }}$ shows that on this space $\mathbf{d} \alpha_{\mu}=\mathbf{d} \alpha_{\xi}$. This is a useful remark since $\mathbf{d} \alpha_{\xi}$ is somewhat easier to compute in examples. We also note, as in an earlier remark, that the magnetic terms can be equivalently computed from the magnetic terms of the $G_{\mu}$ connection rather that the $G$ connection. For instance, for the water molecule, this is easier since in that case, $G=S O(3)$ while $G_{\mu}=S^{1}$.

Let us now introduce a change of variables $r \mapsto T \pi_{Q} \cdot r$ of $\mathcal{S}_{\mathrm{RIG}}$ to $\mathcal{V}_{\mathrm{RIG}}$ and $p \mapsto \mathcal{I} p$ of $\mathcal{W}_{\text {INT }}^{\dagger}$ to $\mathcal{W}_{\text {INT }}^{*}$, so that the representations for $\delta^{2} H_{\xi}\left(z_{e}\right)$ and $\Omega_{z_{e}}$ will be relative to the space $\mathcal{V}_{\text {RIG }} \oplus \mathcal{W}_{\text {INT }} \oplus \mathcal{W}_{\text {INT }}^{*}$. We note at this point that we could have equally well used the representation relative to $\mathcal{V}_{\text {RIG }} \oplus \mathcal{V}_{\text {INT }} \oplus \mathcal{V}_{\text {INT }}^{*}$ and the results below would not materially change (replace $\mathcal{W}$ by $\mathcal{V}$ where appropriate). Using this representation, introduce the following notation for the block diagonal form of $\delta^{2} H_{\xi}:$

$$
\left[\begin{array}{ccc}
A_{\mu} & 0 & 0 \\
0 & \Lambda & 0 \\
0 & 0 & M^{-1}
\end{array}\right]
$$


where $A_{\mu}$ is the co-adjoint orbit block; i.e., the Arnold form, $(2 \times 2$ in the case of $G=S O(3)), \Lambda$ corresponds to the second variation of the amended potential energy, and $M$ corresponds to the metric on the internal variables.

The corresponding symplectic form for the linearized dynamics is

$$
\Omega=\left[\begin{array}{ccc}
L_{\mu} & C & 0 \\
-C^{T} & S & \mathbf{1} \\
0 & -\mathbf{1} & 0
\end{array}\right],
$$

where $S$ is skew-symmetric, $\mathbf{1}$ is the identity and where $C: \mathcal{W}_{\mathrm{INT}} \rightarrow \mathcal{V}_{\mathrm{RIG}}^{*}$ is defined by (2.34). From the earlier remarks, note that in (2.37) and (2.38), the upper block corresponds to the "rotational" dynamics $\left(L_{\mu}\right.$ is in fact the co-adjoint orbit symplectic form for $G$ ) while the two lower blocks correspond to the "internal" dynamics. In (2.38) $C$ represents coupling between the internal and rotational dynamics, while $S$ gives the Coriolis or gyroscopic forces.

The corresponding linearized Hamiltonian vector field is then given by

$$
X_{H}=\left(\Omega^{-1}\right)^{T} \nabla H=\left(\Omega^{-1}\right)^{T} \delta^{2} H_{\xi},
$$

which a computation given below reveals to be

$$
X_{H}(r, q, p)=\left[\begin{array}{ccc}
-L_{\mu}^{-1} A_{\mu} & 0 & -L_{\mu}^{-1} C M^{-1} \\
0 & 0 & M^{-1} \\
-C^{T} L_{\mu}^{-1} A_{\mu} & -\Lambda & -\tilde{S} M^{-1}
\end{array}\right]\left[\begin{array}{c}
r \\
q \\
p
\end{array}\right]
$$

where $\tilde{S}=S+C^{T} L_{\mu}^{-1} C=-\tilde{S}^{T}$.

Thus our linearized equations have the form

$$
\dot{z}=X_{H}(z)
$$

where $z=(r, q, p) \in \mathcal{V}_{\mathrm{RIG}} \times \mathcal{W}_{\mathrm{INT}} \times \mathcal{W}_{\mathrm{INT}}^{*}$ and $X_{H}$ is given by (2.39). See Lewis [1993] for the explicit expression (in terms of the basic data) of the linearized equations. To prove (2.39) we use the first part of the following lemma. (The remainder of the lemma will be used in our stability calculations.)

Lemma 2.11 Consider a vector space $V=V_{1} \oplus V_{2}$ and a linear operator $M: V \rightarrow$ $V^{*}$ given by the partitioned matrix

$$
M=\left[\begin{array}{ll}
B_{11} & B_{12} \\
B_{21} & B_{22}
\end{array}\right]
$$

where $B_{11}: V_{1} \rightarrow V_{1}^{*}, B_{12}: V_{2} \rightarrow V_{1}^{*}, B_{21}: V_{1} \rightarrow V_{2}^{*}$ and $B_{22}: V_{2} \rightarrow V_{2}^{*}$ are linear maps. Assume $B_{11}$ is an isomorphism and let

$$
N=\left[\begin{array}{cc}
B_{11} & 0 \\
0 & B_{22}-B_{21} B_{11}^{-1} B_{12}
\end{array}\right], L=\left[\begin{array}{cc}
\mathbf{1} & 0 \\
-B_{21} B_{11}^{-1} & \mathbf{1}
\end{array}\right], P=\left[\begin{array}{cc}
\mathbf{1} & -B_{11}^{-1} B_{12} \\
0 & \mathbf{1}
\end{array}\right],
$$

so that $N: V \rightarrow V^{*}, L: V^{*} \rightarrow V^{*}$ and $P: V \rightarrow V$. Then 
(i) $L M P=N$

(ii) $M$ is symmetric if and only if $B_{11}$ and $B_{22}$ are symmetric and $B_{12}^{T}=B_{21}$

(iii) If $M$ is symmetric so is $N$

(iv) If $M$ is symmetric then it is positive definite iff $N$ is positive definite; more generally, the signatures of $M$ and $N$ coincide.

Proof (i) is a computation, while (ii) and (iii) are obvious. For (iv), note first that $L^{T}=P$. If $\langle$,$\rangle denotes the natural pairing, then$

$$
\langle L M P x, x\rangle=\left\langle M P x, L^{T} x\right\rangle=\langle M P x, P x\rangle,
$$

which shows that $N$ and $M$ have the same signature since $P$ is invertible.

Lemma 2.12 The linearized Hamiltonian flow with Hamiltonian $\delta^{2} H_{\xi}$ is given by (2.39).

Proof We have $X_{\delta^{2} H_{\xi}}=\left(\Omega^{-1}\right)^{T} \delta^{2} H_{\xi}$. To invert $\Omega$, set $B_{11}=L_{\mu}, B_{12}=\left[\begin{array}{ll}C & 0\end{array}\right], B_{21}=$ $\left[\begin{array}{c}-C^{T} \\ 0\end{array}\right]$, and $B_{22}=\left[\begin{array}{cc}S & \mathbf{1} \\ -\mathbf{1} & 0\end{array}\right]$. From part (i) of Lemma $\mathbf{2 . 1 1}$, we have $M^{-1}=$ $V N^{-1} L$ which gives

$$
\Omega^{-1}=\left[\begin{array}{ccc}
\mathbf{1} & -L_{\mu}^{-1} C & 0 \\
0 & \mathbf{1} & 0 \\
0 & 0 & \mathbf{1}
\end{array}\right]\left[\begin{array}{ccc}
L_{\mu} & 0 & 0 \\
0 & \tilde{S} & \mathbf{1} \\
0 & -\mathbf{1} & 0
\end{array}\right]^{-1}\left[\begin{array}{ccc}
\mathbf{1} & 0 & 0 \\
C^{T} L_{\mu}^{-1} & \mathbf{1} & 0 \\
0 & 0 & \mathbf{1}
\end{array}\right]
$$

where $\tilde{S}=S+C^{T} L_{\mu}^{-1} C=-\tilde{S}^{T}$. Noting that

$$
\left[\begin{array}{cc}
\tilde{S} & \mathbf{1} \\
-\mathbf{1} & 0
\end{array}\right]^{-1}=\left[\begin{array}{cc}
0 & -\mathbf{1} \\
\mathbf{1} & \tilde{S}
\end{array}\right]
$$

we obtain

$$
\Omega^{-1}=\left[\begin{array}{ccc}
L_{\mu}^{-1} & 0 & L_{\mu}^{-1} C \\
0 & 0 & -\mathbf{1} \\
C^{T} L_{\mu}^{-1} & \mathbf{1} & \tilde{S}
\end{array}\right]
$$

and

$$
\left(\Omega^{-1}\right)^{T}=\left[\begin{array}{ccc}
-L_{\mu}^{-1} & 0 & -L_{\mu}^{-1} C \\
0 & 0 & \mathbf{1} \\
-C^{T} L_{\mu}^{-1} & -\mathbf{1} & -\tilde{S}
\end{array}\right]
$$

Hence we get result. 


\section{The Chetaev Function and Lyapunov Instability}

In this section we add a (small) dissipation term to the linear Hamiltonian equation (2.40) and show that this results in Lyapunov instability for the linear system. This is insufficient to prove nonlinear instability of the original system about the given relative equilibrium. For this we prove a result on spectral instability, which we do in $\S 4$.

We add dissipation (damping) to the "internal" variables of the system only, in accordance with the natural physical models. The dissipation is assumed to occur due to the addition, to the Lagrangian, of a Rayleigh dissipation function (see e.g. Whittaker [1959]):

$$
\mathcal{R}=\frac{1}{2} \dot{q}^{T} R \dot{q}=\frac{1}{2}\left(M^{-1} p\right)^{T} R M^{-1} p,
$$

where the Rayleigh dissipation matrix $R: \mathcal{W}_{\mathrm{INT}} \rightarrow \mathcal{W}_{\mathrm{INT}}^{*}$ is symmetric and positive definite: $R=R^{T} \geq 0$.

The system of linearized equations (2.40) becomes

$$
\left.\begin{array}{rl}
\dot{r} & =-L_{\mu}^{-1} A_{\mu} r-L_{\mu}^{-1} C M^{-1} p \\
\dot{q} & =M^{-1} p \\
\dot{p} & =\left(-C^{T} L_{\mu}^{-1} A_{\mu} r-\Lambda q-\tilde{S} M^{-1} p-R M^{-1} p\right) .
\end{array}\right\}
$$

We note that

$$
\frac{d \delta^{2} H_{\xi}}{d t}=-2 \mathcal{R}
$$

The presence of dissipation results in the addition of a term $-R M^{-1}$ to the $(3,3)$ block of the matrix representation (2.39) of the linear system (2.40).

To prove Lyapunov instability, we will employ a generalization of the Chetaev function (Chetaev [1961]; see also Arnold [1987]).

Before doing the general case, it is instructive to analyze the special case $G=S^{1}$, an abelian group, where our system reduces to the form of the system originally analyzed by Chetaev and Thomson. This analysis is relevant, for example, for examining planar rotating systems (see e.g. Oh et al. [1989]).

In this case the $A_{\mu}$ block in (2.37) vanishes and, the linearized flow $X_{\delta^{2} H_{\xi}}$ with the addition of (internal) damping becomes

$$
\left.\begin{array}{c}
\dot{q}=M^{-1} p \\
\dot{p}=\left(-\Lambda q-(S+R) M^{-1} p\right)
\end{array}\right\}
$$


where $R=R^{T} \geq 0$ is the Rayleigh dissipation matrix as above and $S=-S^{T}$ represents the gyroscopic forces in the system.

We shall call (3.4) the Chetaev-Thomson normal form. The example (1.9) analyzed in the introduction is the simplest case of this form.

The basic question addressed by Chetaev is the following. If $\Lambda$ has some negative eigenvalues, yet the spectrum of

$$
\begin{aligned}
& \dot{q}=M^{-1} p \\
& \dot{p}=-\Lambda q-S M^{-1} p
\end{aligned}
$$

is on the imaginary axis, is the system (3.4) unstable? Chetaev showed that this is indeed the case for strong damping; that is, when $R$ is positive definite. Our proof is a slight modification of his. Interestingly, no assumption on $S$ or the the size of $R$ is explicitly needed.

Theorem 3.1 Suppose $\Lambda$ is has one or more negative eigenvalues and $R$ is positive definite. Then the system (3.4) is (Lyapunov) unstable.

The proof is based on the following.

Lemma 3.2 (Lyapunov's Instability Theorem) A linear system is Lyapunov unstable if there is a quadratic function $W$ whose associated quadratic form has at least one negative eigendirection and is such that $\dot{W}$ is negative definite.

See, for example, LaSalle and Lefschetz [1963] for the proof of this lemma.

To utilize this lemma to prove the theorem, we first assume that $\Lambda$ is an isomorphism. Let

$$
W(q, p)=H_{0}(q, p)+\beta B q \cdot M^{-1} p,
$$

where $H_{0}$ is the Hamiltonian for the undamped system, $H_{0}=\frac{1}{2} p^{T} M^{-1} p+\frac{1}{2} q^{T} \Lambda q$, $\beta$ is a scalar, and $B$ is a linear map, both of which are to be determined. Write

$$
W=\frac{1}{2}\left[p^{T} q^{T}\right]\left[\begin{array}{cc}
M^{-1} & \beta\left(M^{T}\right)^{-1} B \\
\beta B^{T} M^{-1} & \Lambda
\end{array}\right]\left[\begin{array}{l}
p \\
q
\end{array}\right] .
$$

Calculating the time derivative, we find that

$$
\begin{aligned}
& \dot{W}=-\left(p^{T} q^{T}\right) \times \\
& {\left[\begin{array}{cc}
\left(M^{T}\right)^{-1} R M^{-1} & \frac{\beta}{2}\left(M^{T}\right)^{-1}(R-S) M^{-1} B \\
-\frac{\beta}{2}\left(\left(M^{T}\right)^{-1} B M^{-1}+\left(M^{T}\right)^{-1} B^{T} M^{-1}\right) & \\
\frac{\beta}{2} B^{T}\left(M^{T}\right)^{-1}(R+S) M^{-1} & \frac{\beta}{2}\left(B^{T} M^{-1} \Lambda+\Lambda\left(M^{T}\right)^{-1} B\right)
\end{array}\right]\left[\begin{array}{c}
p \\
q
\end{array}\right] .}
\end{aligned}
$$


Now choose any positive definite symmetric map $K: \mathcal{W}_{\text {INT }} \rightarrow \mathcal{W}_{\text {INT }}^{*}$. Our choice of $B$ will depend on $K$, but $K$ may be chosen arbitrarily, and this freedom will be important below. We let

$$
B=M^{T} K^{-1} \Lambda: \mathcal{W}_{\mathrm{INT}} \rightarrow \mathcal{W}_{\mathrm{INT}}^{*} .
$$

From Lemma 2.11, we see that $\dot{W}$ is negative definite if and only if

$$
\left[\begin{array}{cc}
\left(M^{T}\right)^{-1} R M^{-1}+O(\beta) & 0 \\
0 & \beta \Lambda K^{-1} \Lambda+O\left(\beta^{2}\right)
\end{array}\right]
$$

is positive definite. This is clearly true for $\beta>0$ sufficiently small since $\left(M^{T}\right)^{-1} R M^{-1}$ and $\Lambda K^{-1} \Lambda$ are symmetric and postive definite. On the other hand, by a similar argument, $W$ has at least one negative eigendirection for $\beta$ sufficiently small. Hence by lemma 3.2 , we have instability.

To prove the general case, in which $\Lambda$ is allowed to be degenerate, we proceed as follows. Split the space

$$
\mathcal{W}_{\mathrm{INT}}=\operatorname{ker} \Lambda \oplus(\operatorname{ker} \Lambda)^{\perp}
$$

into the direct sum of the kernel of $\Lambda$ and its orthogonal complement in the inner product corresponding to $M$. This induces a similar decompostion of the dual spaces using $M$ as an isomorphism. Denote with a subscript 1 the first component in this decomposition and with a subscript 2 , the second component. In this decomposition, we have the block structure

$$
\Lambda=\left[\begin{array}{cc}
0 & 0 \\
0 & \Lambda_{2}
\end{array}\right]
$$

and

$$
M=\left[\begin{array}{cc}
M_{1} & 0 \\
0 & M_{2}
\end{array}\right]
$$

The equations (3.4) in this splitting become

$$
\left.\begin{array}{c}
\dot{q}_{1}=M_{1}^{-1} p_{1} \\
\dot{q}_{2}=M_{2}^{-1} p_{2} \\
\dot{p}=\left(\left(0,-\Lambda_{2} q_{2}\right)-(S+R) M^{-1} p\right) .
\end{array}\right\}
$$

Notice that the first equation for $q_{1}$ decouples from the next three equations. Now we proceed as above, with the function $W(q, p)$ replaced by the following function of $\left(q_{2}, p\right)$ :

$$
W\left(q_{2}, p\right)=\frac{1}{2} p^{T} M^{-1} p+\frac{1}{2} q_{2}^{T} \Lambda q_{2}+\beta M_{2}^{T} K_{2}^{-1} \Lambda_{2} q_{2} \cdot\left(M_{2}^{T}\right)^{-1} p_{2},
$$

where $\left.K_{2}:(\operatorname{ker} \Lambda)^{\perp} \rightarrow M(\operatorname{ker} \Lambda)^{\perp}\right) \subset \mathcal{W}_{\text {INT }}^{*}$ is positive definite symmetric. Note especially that here we are using our freedom to choose $K$; in Chetaev, the initial 
choice $K=\Lambda$ was made, which required $\Lambda$ to be invertible. We now compute $\dot{W}$ as above, and obtain an expression similar to (3.7) but with the blocks done according to the variables $\left(p, q_{2}\right)$, and in which the top right and lower left expressions are modified, but are still multiplied by $\beta$, and where the lower right hand block is replaced by the expression $\beta \Lambda_{2} K_{2}^{-1} \Lambda_{2}$. Now repeat the argument above.

We now extend our analysis to the general equation (2.40) i.e., to an arbitrary nonabelian symmetry group $G$. We show that indefiniteness of $\delta^{2} H_{\xi}$ at a given relative equilibrium implies Lyapunov instability (again spectral instability follows from the analysis in $\S 4$ ).

The main ingredient is a generalization of the Chetaev function (3.5). As above, we establish definiteness of the time derivative of the function, but the analysis is now more complex. Also we need an assumption on the coupling matrix $C$ between the internal and rotational modes.

Theorem 3.3 Suppose $A_{\mu}$ is nondegenerate and either $\Lambda$ or $A_{\mu}$ has at least one negative eigenvalue. Suppose that $R>0$ and that $C^{T}$ is injective. Then the system (3.2) is Lyapunov unstable.

Proof As in the abelian case, we start with the assumption that $\Lambda$ is an isomorphism. In this case, let

$$
\begin{aligned}
W(q, p, r)= & \frac{1}{2} p \cdot M^{-1} p+\frac{1}{2} q \cdot \Lambda q+\frac{1}{2} r \cdot A_{\mu} r \\
& +\beta B q \cdot M^{-1} p+\alpha D r \cdot M^{-1} p+\gamma E r \cdot \Lambda q
\end{aligned}
$$

where $\alpha, \beta$, and $\gamma$ are scalars and $B, D$, and $E$ are linear operators, all to be chosen. We write the matrix representation of $W$, in the ordering $(p, q, r)$ as :

$$
W=\frac{1}{2}\left[\begin{array}{ccc}
M^{-1} & \frac{\beta}{2}\left(M^{T}\right)^{-1} B & \frac{\alpha}{2}\left(M^{T}\right)^{-1} D \\
\frac{\beta}{2} B^{T} M^{-1} & \Lambda & \frac{\gamma}{2} \Lambda E \\
\frac{\alpha}{2} D^{T} M^{-1} & \frac{\gamma}{2} E^{T} \Lambda & A_{\mu}
\end{array}\right] .
$$


After a lengthy computation, we find that the matrix representation of $-\dot{W}$ is:

$$
\begin{array}{ccc}
\left(M^{T}\right)^{-1} R M^{-1} & & \\
-\frac{\beta}{2}\left(\left(M^{T}\right)^{-1} B^{T} M^{-1}\right. & \frac{\beta}{2}\left(M^{T}\right)^{-1}(R-\tilde{S}) M^{-1} B & \frac{\alpha}{2}\left(M^{T}\right)^{-1}(R-\tilde{S}) M^{-1} D \\
\left.+\left(M^{T}\right)^{-1} B M^{-1}\right) & +\frac{\alpha}{2} M^{-1} D L_{\mu}^{-1} A_{\mu} \\
+\frac{\alpha}{2}\left(-\left(M^{T}\right)^{-1} C^{T} L_{\mu}^{-1} D^{T} M^{-1}\right. & -\frac{\gamma}{2}\left(M^{T}\right)^{-1} C^{T} L_{\mu}^{-1} E^{T} \Lambda & -\frac{\gamma}{2}\left(M^{T}\right)^{-1} \Lambda E \\
\left.+\left(M^{T}\right)^{-1} D L_{\mu}^{-1} C M^{-1}\right) & & \\
& & \\
\left(\frac{\beta}{2}\left(M^{T}\right)^{-1}(R-\tilde{S}) M^{-1} B\right. & \frac{\beta}{2}\left(B^{T}\left(M^{T}\right)^{-1} \Lambda\right. & \frac{\alpha}{2} \Lambda M^{-1} D \\
\left.-\frac{\gamma}{2}\left(M^{T}\right)^{-1} C^{T} L_{\mu}^{-1} E^{T} \Lambda\right)^{T} & \left.+\Lambda M^{-1} B\right) & +\frac{\beta}{2} B^{T}\left(M^{T}\right)^{-1} C^{T} L_{\mu}^{-1} A_{\mu} \\
& & +\frac{\gamma}{2} \Lambda E L_{\mu}^{-1} A_{\mu} \\
\left(\frac{\alpha}{2}\left(M^{T}\right)^{-1}(R-\tilde{S}) M^{-1} D\right. & \left(\frac{\alpha}{2} \Lambda M^{-1} D\right. & \\
+\frac{\alpha}{2} M^{-1} D L_{\mu}^{-1} A_{\mu} & +\frac{\beta}{2} B^{T}\left(M^{T}\right)^{-1} C^{T} L_{\mu}^{-1} A_{\mu} & \frac{\alpha}{2}\left(D^{T} M^{-1} C^{T} L_{\mu}^{-1} A_{\mu}\right. \\
\left.-\frac{\gamma}{2}\left(M^{T}\right)^{-1} \Lambda E\right)^{T} & \left.+\frac{\gamma}{2} \Lambda E L_{\mu}^{-1} A_{\mu}\right)^{T} & \left.-A_{\mu} L_{\mu}^{-1} C\left(M^{T}\right)^{-1} D\right)
\end{array}
$$

We now show that $-\dot{W}$ is positive definite for suitable choices of $\alpha, \beta, \gamma, B, D$, and $E$. To do this we block diagonalize $-\dot{W}$ by repeated applications of lemma 2.11 . Write $-\dot{W}$ in its partitioned form as

$$
-\dot{W}=\left[\begin{array}{ccc}
A_{11} & A_{12} & A_{13} \\
A_{12}^{T} & A_{22} & A_{23} \\
A_{13}^{T} & A_{23}^{T} & A_{33}
\end{array}\right] .
$$

Then

$$
\begin{gathered}
{\left[\begin{array}{ccc}
\mathbf{1} & 0 & 0 \\
-A_{12}^{T} A_{11}^{-1} & \mathbf{1} & 0 \\
0 & 0 & \mathbf{1}
\end{array}\right]\left[\begin{array}{ccc}
A_{11} & A_{12} & A_{13} \\
A_{12}^{T} & A_{22} & A_{23} \\
A_{13}^{T} & A_{23}^{T} & A_{33}
\end{array}\right]\left[\begin{array}{ccc}
\mathbf{1} & -A_{11}^{-1} A_{12} & 0 \\
0 & \mathbf{1} & 0 \\
0 & 0 & \mathbf{1}
\end{array}\right]} \\
=\left[\begin{array}{ccc}
A_{11} & 0 & A_{13} \\
0 & A_{22}-A_{12}^{T} A_{11}^{-1} A_{12} & -A_{12}^{T} A_{11}^{-1} A_{13}+A_{23} \\
A_{13}^{T} & -A_{13}^{T} A_{11}^{-1} A_{12}+A_{23}^{T} & A_{33}
\end{array}\right] .
\end{gathered}
$$

Multiplying (3.11) by

$$
\left[\begin{array}{ccc}
\mathbf{1} & 0 & -A_{11}^{-1} A_{13} \\
0 & \mathbf{1} & 0 \\
0 & 0 & \mathbf{1}
\end{array}\right]
$$


on the right and by the transpose of (3.12) on the left yields

$$
\left[\begin{array}{ccc}
A_{11} & 0 & 0 \\
0 & A_{22}-A_{12}^{T} A_{11}^{-1} A_{12} & -A_{12}^{T} A_{11}^{-1} A_{13}+A_{23} \\
0 & -A_{13}^{T} A_{11}^{-1} A_{12}+A_{23}^{T} & -A_{13}^{T} A_{11}^{-1} A_{13}+A_{33}
\end{array}\right] .
$$

Then a final application of the lemma to (3.13) yields the block diagonal form

$$
\left[\begin{array}{ccc}
A_{11} & 0 & 0 \\
0 & A_{22}-A_{12}^{T} A_{11}^{-1} A_{12} & 0 \\
0 & 0 & \tilde{A}_{33}
\end{array}\right]
$$

where

$$
\begin{aligned}
\tilde{A}_{33}= & A_{33}-A_{13}^{T} A_{11}^{-1} A_{13}-\left(A_{23}^{T}-A_{13} A_{11}^{-1} A_{12}\right) \times \\
& \left(A_{22}-A_{12}^{T} A_{11}^{-1} A_{12}\right)^{-1}\left(A_{23}-A_{12}^{T} A_{11}^{-1} A_{13}\right) .
\end{aligned}
$$

Now $A_{11}$ in (3.10) is positive definite if $\alpha$ and $\beta$ are small, since $R$ is positive definite. Choose, as in Theorem 3.1, $B=M K^{-1} \Lambda$, and assume that $\gamma$ is small, then $A_{22}-A_{12}^{T} A_{11}^{-1} A_{12}=\beta \Lambda K^{-1} \Lambda-A_{12}^{T} A_{11}^{-1} A_{12}$. Since the second term is of higher order in $\alpha, \beta, \gamma$, this is positive definite. It remains to prove positive definiteness of $\tilde{A}_{33}$. Firstly, choose

$$
D=M^{T} K^{-1} C^{T} L_{\mu}^{-1} A_{\mu}: \mathcal{S}_{\mathrm{RIG}} \rightarrow \mathcal{W}_{\mathrm{INT}}^{*}
$$

Then we find:

$$
\begin{aligned}
A_{11}= & \left(M^{T}\right)^{-1} R M^{-1}+O(\alpha)+O(\beta) \\
A_{12}= & \frac{\beta}{2}\left(M^{T}\right)^{-1}(R-\tilde{S}) K^{-1} \Lambda-\frac{\gamma}{2}\left(M^{T}\right)^{-1} C^{T} L_{\mu}^{-1} E^{T} \Lambda \\
A_{13}= & \frac{\alpha}{2}\left(M^{T}\right)^{-1}(R-\tilde{S}) K^{-1} C^{T} L_{\mu}^{-1} A_{\mu}+\frac{\alpha}{2} M^{-1} M K^{-1} C^{T} L_{\mu}^{-1} A_{\mu} L_{\mu}^{-1} A_{\mu} \\
& -\frac{\gamma}{2}\left(M^{T}\right)^{-1} \Lambda E \\
A_{23}= & \left(\frac{\alpha}{2}+\frac{\beta}{2}\right) \Lambda K^{-1} C^{T} L_{\mu}^{-1} A_{\mu}+\frac{\gamma}{2} \Lambda E L_{\mu}^{-1} A_{\mu} \\
A_{22}= & \beta \Lambda K^{-1} \Lambda \\
A_{33}= & \alpha\left(C^{T} L_{\mu}^{-1} A_{\mu}\right)^{T} K^{-1}\left(C^{T} L_{\mu}^{-1} A_{\mu}\right) .
\end{aligned}
$$

To show that $\tilde{A}_{33}$ is positive definite, we set $\alpha=\beta=\gamma$ and write it as a term linear in $\alpha$ plus higher order terms in $\alpha$. Then we show the term linear in $\alpha$ is indeed positive definite for $C^{T}$ injective and a suitable choice of $E$.

We now isolate the terms in $\tilde{A}_{33}$ that are linear in $\alpha$. Since

$$
A_{11}=\left(M^{T}\right)^{-1} R M^{-1}+O(\alpha)
$$


we get $A_{11}^{-1}=M R^{-1} M(1+O(\alpha))$. Also $A_{13}, A_{23}$ and $A_{33}$ are all $O(\alpha)$. Hence

$$
A_{13}^{T} A_{11}^{-1} A_{13}=O\left(\alpha^{2}\right)
$$

and so does not affect definiteness, for small $\alpha$. Next,

$$
A_{22}-A_{12}^{T} A_{11}^{-1} A_{12}=\alpha \Lambda K^{-1} \Lambda+O\left(\alpha^{2}\right)=\alpha \Lambda K^{-1} \Lambda(1+O(\alpha))
$$

and so

$$
\left(A_{22}-A_{12}^{T} A_{11}^{-1} A_{12}\right)^{-1}=\frac{1}{\alpha} \Lambda^{-1} K \Lambda^{-1}(1+O(\alpha))
$$

Also,

$$
A_{23}-A_{12}^{T} A_{11}^{-1} A_{13}=A_{23}+O\left(\alpha^{2}\right)
$$

and thus

$$
\begin{aligned}
\tilde{A}_{33}= & A_{33}+O\left(\alpha^{2}\right)-\left(A_{23}^{T}+O\left(\alpha^{2}\right)\right) \frac{1}{\alpha} \Lambda^{-1} K \Lambda^{-1}(1+O(\alpha))\left(A_{23}+O\left(\alpha^{2}\right)\right) \\
= & A_{33}+O\left(\alpha^{2}\right)-\frac{1}{\alpha}\left(A_{23}^{T} \Lambda^{-1} K \Lambda^{-1}+O\left(\alpha^{2}\right)+A_{23}^{T} \Lambda^{-1} K \Lambda^{-1} O(\alpha)\right. \\
& \left.+O\left(\alpha^{3}\right)\right)\left(A_{23}+O\left(\alpha^{2}\right)\right) \\
= & A_{33}-\frac{1}{\alpha} A_{23}^{T} \Lambda^{-1} K \Lambda^{-1} A_{23}+O\left(\alpha^{2}\right) .
\end{aligned}
$$

Hence the term in $\tilde{A}_{33}$ linear in $\alpha$ is given by

$$
\begin{gathered}
\alpha\left(C^{T} L_{\mu}^{-1} A_{\mu}\right)^{T} K^{-1}\left(C^{T} L_{\mu}^{-1} A_{\mu}\right) \\
+\frac{1}{\alpha}\left(\alpha A_{\mu} L_{\mu}^{-1} C K^{-1} \Lambda+\frac{1}{2} \alpha A_{\mu} L_{\mu}^{-1} E^{T} \Lambda\right) \Lambda^{-1} K \Lambda^{-1}\left(\alpha \Lambda K^{-1} C^{T} L_{\mu}^{-1} A_{\mu}+\frac{1}{2} \alpha \Lambda E L_{\mu}^{-1} A_{\mu}\right) \\
=\left(C^{T} L_{\mu}^{-1} A_{\mu}\right)^{T} K^{-1}\left(C^{T} L_{\mu}^{-1} A_{\mu}\right)+A_{\mu} L_{\mu}^{-1} C K^{-1} C^{T} L_{\mu}^{-1} A_{\mu} \\
+\frac{1}{2} \alpha A_{\mu} L_{\mu}^{-1} E^{T} C^{T} L_{\mu}^{-1} A_{\mu}+\frac{1}{2} \alpha A_{\mu} L_{\mu}^{-1} C E L_{\mu}^{-1} A_{\mu} \\
+\frac{1}{4} \alpha A_{\mu} L_{\mu}^{-1} E^{T} K E L_{\mu}^{-1} A_{\mu} .
\end{gathered}
$$

Since $\left(A_{\mu} L_{\mu}^{-1}\right)=-\left(L_{\mu}^{-1} A_{\mu}\right)^{T}$, the first two terms cancel and we obtain

$$
-\frac{\alpha}{2}\left(L_{\mu}^{-1} A_{\mu}\right)^{T}\left[C E+(C E)^{T}+\frac{1}{2} E^{T} K E\right]\left(L_{\mu}^{-1} A_{\mu}\right) .
$$

Now let $E=-K^{-1} C^{T}$. Then $C E+(C E)^{T}+\frac{1}{2} E^{T} K E=-\frac{3}{2} C K^{-1} C^{T}$ and hence

$$
\tilde{A}_{33}=\frac{3 \alpha}{4}\left(L_{\mu}^{-1} A_{\mu}\right)^{T}\left(C K^{-1} C^{T}\right)\left(L_{\mu}^{-1} A_{\mu}\right)+O\left(\alpha^{2}\right)
$$


which is positive definite since $C^{T}$ is injective and $\alpha>0$.

Since $W$ is clearly indefinite and $\dot{W}$ is negative definite, we have Lyapunov instability by Lyapunov's instability theorem.

To prove the theorem in the case that $\Lambda$ is degenerate, split the variable $q$ into $\left(q_{1}, q_{2}\right)$ as in the proof of the abelian case and note that the equations (3.2) decouple into equations for $q_{1}$ and $\left(r, p, q_{2}\right)$. Now repeat the argument using the same modifications as in the abelian case.

For completeness, we give the details in the extreme case $\Lambda=0$. In this case, the linearized dynamics with added dissipation in the block-diagonal normal form takes the following "triangular" form:

$$
\left.\begin{array}{rl}
M \dot{q} & =p \\
\dot{p} & =-(\tilde{S}+R) M^{-1} p-C^{T} L_{\mu}^{-1} A_{\mu} r \\
\dot{r} & =-L_{\mu}^{-1} A_{\mu}^{-1} r-L_{\mu}^{-1} C M^{-1} p,
\end{array}\right\}
$$

where $\tilde{S}=S+C^{T} L_{\mu}^{-1} C$, and $R=R^{T} \geq 0$ is a matrix of damping coefficients. Note that projecting out the shape variable $q$ leaves the reduced system from (3.18) involving $\dot{p}$ and $\dot{r}$ only, which can be handled separately. Let

$$
W(p, r)=\frac{1}{2} p \cdot M^{-1} p+\frac{1}{2} r \cdot A_{\mu} r+\alpha D r \cdot M^{-1} p
$$

where $\alpha$ is a scalar and $D$ is to be chosen. We will show that $\alpha$ and $D$ can be so chosen that $W(p, r)$ is a Chetaev function for the reduced system-the second and third equations of (3.18) i.e., $W(p, r)$ is indefinite and its total derivative $\dot{W}$ along trajectories of (3.18) is negative definite. This would then establish the Lyapunov instability of the reduced system and consequently of the full system (3.18). As above, choose

$$
D=M K^{-1} C^{T} L_{\mu}^{-1} A_{\mu}
$$

It is then easy to verify that

$$
\dot{W}=-\left[p^{T} r^{T}\right]\left[\begin{array}{ll}
Q_{11} & Q_{12} \\
Q_{12}^{T} & Q_{22}
\end{array}\right]\left[\begin{array}{l}
p \\
r
\end{array}\right]
$$

where,

$$
\begin{aligned}
Q_{11} & =\left(M^{T}\right)^{-1} R M^{-1}+O(\alpha) \\
Q_{12} & =\frac{\alpha}{2}\left(\left(M^{T}\right)^{-1}(R-\tilde{S}) K^{-1} C^{T} L_{\mu}^{-1} A_{\mu}+K^{-1} C^{T} L_{\mu}^{-1} A_{\mu} L_{\mu}^{-1} A_{\mu}\right) \\
Q_{22} & =\alpha\left(C^{T} L_{\mu}^{-1} A_{\mu}\right)^{T} K^{-1}\left(C^{T} L_{\mu}^{-1} A_{\mu}\right) .
\end{aligned}
$$


By hypothesis $Q_{22}>0$. As above, there is a range of $\alpha$ for which the matrix

$$
Q_{\alpha}=\left[\begin{array}{ll}
Q_{11} & Q_{12} \\
Q_{12}^{T} & Q_{22}
\end{array}\right]
$$

is positive definite. Further, since the signature of a hyperbolic matrix is invariant under small perturbations, one can further choose $\alpha$ in the range $(0, c)$ such that,

$$
\text { signature }\left[\begin{array}{cc}
M^{-1} & \frac{\alpha}{2} M^{-1} D \\
\frac{\alpha}{2} D^{T} M^{-1} & A_{\mu}
\end{array}\right]=\text { signature }\left[\begin{array}{cc}
M^{-1} & 0 \\
0 & A_{\mu}
\end{array}\right] \text {. }
$$

The matrix $\left[\begin{array}{cc}M^{-1} & 0 \\ 0 & A_{\mu}\end{array}\right]$ is indefinite by hypothesis. Thus we have a range of $\alpha$ for which $W$ is a Chetaev function and we have proved Lyapunov instability.

Remark We leave it to the reader to verify that standard eigenvalue inequalities lead to the condition,

$$
0<\alpha<c
$$

where

$$
\begin{aligned}
c= & \min \left\{c_{1}, c_{2}^{-1}\right\}, \\
c_{1}= & \left\{\begin{array}{lll}
\infty & \text { if } & \lambda_{\min }\left(C^{T} L_{\mu}^{-1} A_{\mu} L_{\mu}^{-1} C\right) \geq 0 \\
\frac{\lambda_{\min }\left(M^{-1} R M^{-1}\right)}{\left|\lambda_{\min }\left(C^{T} L_{\mu}^{-1} A_{\mu} L_{\mu}^{-1} C\right)\right|} & \text { if } & \lambda_{\min }\left(C^{T} L_{\mu}^{-1} A_{\mu} L_{\mu}^{-1} C\right)<0,
\end{array}\right. \\
c_{2}= & \left\|\left(M^{-1} R M^{-1}\right)^{-1 / 2} C^{T} L_{\mu}^{-1} A_{\mu} L_{\mu}^{-1} C\left(M^{-1} R M^{-1}\right)^{-1 / 2}\right\| \\
& +\lambda_{\max }\left(Q_{12}^{T} M R^{-1} M Q_{12}\right) / \lambda_{\min }\left(Q_{22}\right)
\end{aligned}
$$

and $\|\cdot\|$ denotes the Euclidean norm.

An illustration of the instability result of Theorem 3.3 in the case of $\Lambda=0$, and of the effective use of the block diagonal normal form will be given in the examples in $\S 6$.

\section{Instability of Relative Equilibria}

Our main result shows that if $\delta^{2} H_{\xi}$ is indefinite at a given relative equilibrium, the system is dissipation unstable about that equilibrium. To do this, it is sufficient to prove spectral instability of the linear system (3.2). In $\S \mathbf{3}$ we proved Lyapunov instability of this system. As discussed in the introduction, this is not sufficient to prove instability of the nonlinear system. Hence we need to show that we do in fact have spectral instability. This will follow from the following proposition which utilizes the eigenstructure of the linearized Hamiltonian system (i.e., with $R=0$ ) and a key observation of Hahn [1967]. 


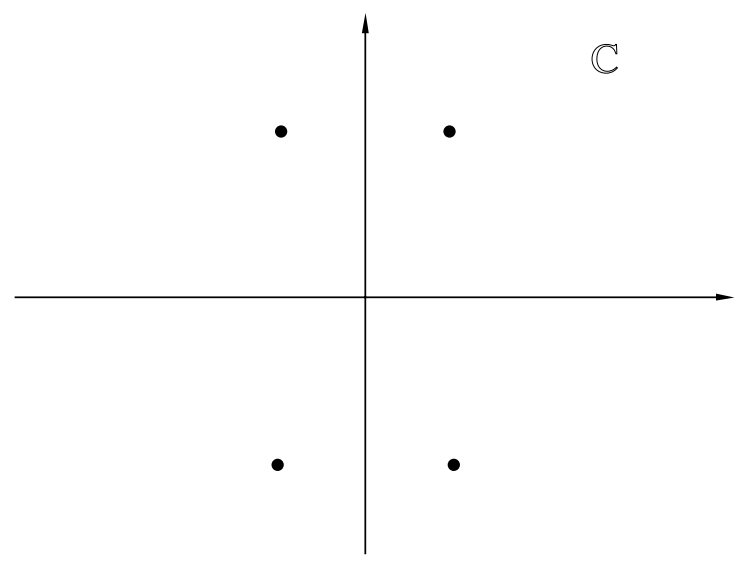

Figure 1: The case of an eigenvalue quadruplet.

Proposition 4.1 Let $\dot{x}=X_{H}(x)$ be a linear Hamiltonian system. Suppose that one adds a small linear perturbation to $X_{H}$ (in particular, a damping term) and that for the augmented system there exists a quadratic form $W$ which has at least one negative eigendirection and which satisfies $\dot{W}<0$ along the flow. Then the augmented system is spectrally unstable.

Proof The properties of $W$ imply that the augmented system is Lyapunov unstable, as we have seen. We now show that it is spectrally unstable. Henceforth, we shall refer to the augmented system as the damped system and the perturbation as damping.

We consider firstly the eigenvalue configurations of the undamped linear Hamiltonian system. From the general properties of Hamiltonian matrices (see e.g. Abraham and Marsden [1978]) the possible configurations can be grouped into the following four categories:

1. There is at least one quadruplet, i.e., an eigenvalue configuration shown in Figure 4.1:

2. There is at least one pair of real eigenvalues, as in Figure 4.2.

3. Neither 1. nor 2. holds but all the eigenvalues are on the imaginary axis and are simple.

4. All the eigenvalues are on the imaginary axis and there is at least one multiple eigenvalue.

Now add the damping terms. In cases 1 . and 2., small damping leaves eigenvalues in the right half plane. Hence we have spectral instability. Now consider case 3. All eigenvalues cannot move to the left half plane since this implies Lyapunov stability and we have instability. They cannot all remain on the imaginary axis since (for small damping) they remain distinct and hence all solutions would be periodic and hence stable. Similarly if some move into the left half plane and some remain (distinct) on the imaginary axis, the system is still stable. The only remaining 


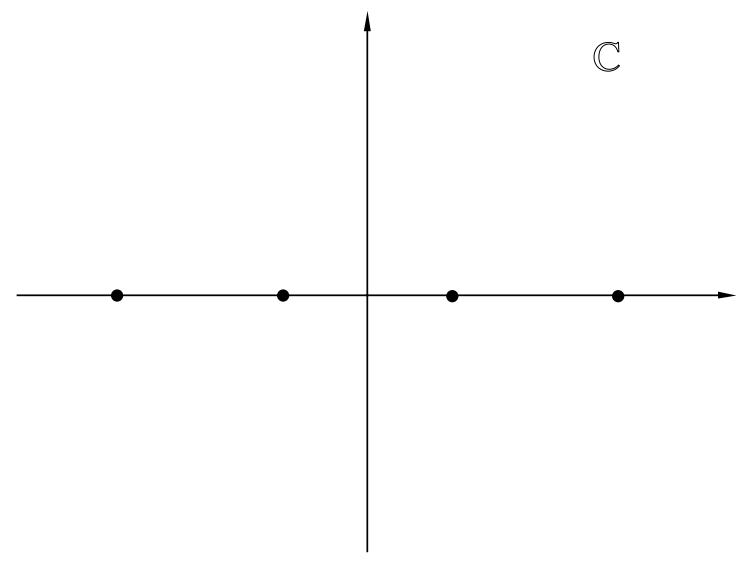

Figure 2: The case of eigenvalues on the real axis.

possibility is at least one moves to the right half plane and we thus have spectral instability.

Finally consider case 4 . If any eigenvalues move into the right half plane we have spectral instability and are done. Now if all eigenvalues moved to the left half plane the system would be stable and we know it is unstable. Similarly it is impossible for some to move to the left and for those that remain on the imaginary axis to be simple, for this again implies stability.

The only remaining possibilities are a multiple zero eigenvalue or a multiple pair of conjugate purely imaginary eigenvalues remaining on the imaginary axis after the addition of damping. We can show that both situations are impossible for they contradict $\dot{W}<0$ :

Suppose firstly that there is a zero eigenvalue. Let $W=z^{T} Q z$ and $X_{H}(z)=A z$. Then $\dot{W}=z^{T}\left(A^{T} Q+Q A\right) z$. But there exists an $\tilde{z} \neq 0$ and that $A \tilde{z}=0$ and here $\dot{W}(\tilde{z})=0$, contradicting $\dot{W}<0$.

Now suppose there is a pair of conjugate purely imaginary multiple eigenvalues. Then there exists an invariant subspace for the flow, which is a subspace of the generalized eigenspace corresponding to the multiple eigenvalues, which is invariant for the matrix

$$
\left[\begin{array}{cccc}
0 & -b & 0 & 0 \\
b & 0 & 0 & 0 \\
1 & 0 & 0 & -b \\
0 & 1 & b & 0
\end{array}\right]
$$

Now we can use the following argument of Hahn [1967]. There exists a solution of the system corresponding to (4.1) of the form

$$
z_{1}=z_{2}=0 \quad z_{3}=\cos b t \quad z_{4}=\sin b t .
$$


However, $W$ is a periodic function of $t$ when evaluated on the above solution. On the other hand,

$$
W(t)=W_{0}+\int_{t_{0}}^{t} \dot{W}(s) d s .
$$

Since $\dot{W}<0,|\dot{W}|$ is bounded away from zero on this curve. Hence the integral would not be bounded as $t \rightarrow \infty$ and $W$ cannot be periodic on this trajectory. Hence we cannot have a pair of conjugate purely imaginary multiple eigenvalues since this contradicts $\dot{W}<0$.

Thus we see that at least one eigenvalue must be in the right half plane and the system is spectrally unstable.

Combining our results and using the notation of $\S \mathbf{2}$, we get

Theorem 4.2 Assume (for non-abelian groups) $C^{T}$ is injective, and the second variation of the Energy-Momentum function $H_{\xi}$ of the Hamiltonian system is indefinite at a given relative equilibrium. Then the addition of small strong (internal) Rayleigh dissipation gives spectral instability of the system about that relative equilibrium.

The arguments we have given are designed especially to be applicable to infinite dimensional systems, even though we have so far confined our attention to finite dimensional ones. There need to be appropriate assumptions on the semigroups involved, and assumptions on the spectra, but it seems that the main assumption needed for the above analysis to be valid is that the spectrum of the unperturbed problem be discrete, with eigenvalues having at most finite multiplicity.

Two interesting problems are the whirling string and the rotating circular liquid drop. We hope to pursue the analysis of these problems using the present techniques in another publication. We analyze a simple rotating beam, where the infinitedimensional calculation reduces to a finite-dimensional one, in $\S \mathbf{6}$.

We now make some remarks on the condition requiring $C^{T}$ to be injective.

\section{Remarks}

1 Since $C^{T}: \mathcal{V}_{\mathrm{RIG}} \rightarrow \mathcal{W}_{\mathrm{INT}}^{*}$, note it can be injective only if $\operatorname{dim} \mathcal{V}_{\mathrm{RIG}}=\operatorname{dim} \mathfrak{g}_{\mu_{e}}^{\perp} \leq$ $\operatorname{dim} Q-\operatorname{dim} G$, i.e., $\operatorname{dim} \mathcal{O}_{\mu_{e}} \leq \operatorname{dim}(Q / G)$. For example, for $S O(3)$, this says that $5 \leq \operatorname{dim} Q$. For a rigid body with rotors and $G=S O(3)$, this says that there must be at least two rotors.

2 We claim that $C^{T}$ is injective, i.e., $C$ is surjective, if

$$
\mathcal{V}_{\mathrm{INT}}^{\perp} \cap \mathfrak{g} \cdot q_{e}=\{0\} .
$$

Proof From (2.34),

$$
\left\langle C^{T}(\Delta q), \delta q\right\rangle=-\left\langle\left\langle\zeta_{Q}\left(q_{e}\right), \delta q\right\rangle\right\rangle .
$$


Suppose this is zero for all $\delta q \in \mathcal{V}_{\text {INT. }}$. Then $\zeta_{Q}\left(q_{e}\right) \in \mathcal{V}_{\text {INT }}^{\perp}$ and so by hypothesis, $\zeta_{Q}\left(q_{e}\right)=0$. By freeness, $\zeta=0$, and so by Lemma 2.7, $\zeta=\mathbb{I}\left(q_{e}\right)^{-1} \operatorname{ad}_{\eta}^{*} \mu$, where $\eta \in \mathfrak{g}_{\mu_{e}}$, and so as $\eta \in \mathfrak{g}_{\mu_{e}}^{\perp}$, by (2.22), $\eta=0$, and so by (2.32), $\Delta q=0$.

Notice that the above condition is a hypothesis on $\mathcal{V}_{\text {INT }}$ being "genuinely different" from the "naive" choice of internal space, namely $\left[\mathfrak{g} \cdot q_{e}\right]^{\perp}$.

Remark on Internal Symmetries: In some situations, we will have internal symmetries in the system. In the Hamiltonian case each such symmetry would enable one to reduce the system by one degree of freedom. In the presence of damping (dissipation) we cannot of course do this, but one can nonetheless eliminate the corresponding configuration variables. This ensures that the matrix representation of $\dot{W}$ will be negative semi-definite rather than definite (with zero eigenvalues due to the symmetry). The same analysis as before then applies. This situation will be illustrated in $\S 6$.

\section{Dissipation-Induced Movement of Eigenvalues}

In contrast with the method of Routh-Hurwitz that requires explicit calculations with characteristic polynomials, the methods of the present paper allow one to predict dissipation-induced instability of relative equilibria solely on the basis of signature computations -indefiniteness of $\delta^{2} H_{\xi}$ and injectivity of $C^{T}$. In this sense, the present paper is closer in spirit to the work of Hermite on Hankel quadratic forms, cf. the last chapter of vol 2. of Gantmacher [1959]. However, the classical work of Routh, Hermite and Hurwitz was aimed at getting more refined informationsuch as the number of right half plane eigenvalues - than just predicting instability. In the present context, a closely related question is that of determining speeds of crossing (into the right half plane) of pure imaginary eigenvalues due to dissipative perturbations of an underlying Hamiltonian system. In this section we discuss some formulae to compute such speeds and thereby track in detail the mechanism of instability. Our formulae generalize the previous work of Krein [1950] and McKay [1991], and when specialized to the block-diagonal normal form (abelian as well as non-abelian cases) yield new and explicit formulae for crossing speeds.

Keeping in mind the well-known connections between the asymptotic stability of a linear system and solutions to the matrix Lyapunov equation $c f$. Bellman [1963], Brockett [1970], Taussky [1961], our proofs will have a definite Lyapunov theory flavor. In particular, we will not need the Kato perturbation lemma $c f$. McKay [1991]. We also refer to Kirk, Marsden and Silber [1996] for an alternative argument.

We first prove a basic result.

Lemma 5.1 Consider a linear system $\dot{x}=A x$ and a quadratic form $V(x)=$ $\frac{1}{2} x^{T} Q x$. Let $\dot{V}(x)$ denote the total derivative of $V$ along trajectories of the linear system, evaluated at $x$. Let $\lambda=\lambda_{r}+i \lambda_{i} \in \operatorname{spectrum}(A)$. Let $\xi=x_{r}+i x_{i}$ denote an eigenvector of $A$ corresponding to $\lambda$. Then, 


$$
\lambda_{r}=\frac{\dot{V}\left(x_{r}\right)+\dot{V}\left(x_{i}\right)}{2\left(V\left(x_{r}\right)+V\left(x_{i}\right)\right)} .
$$

Proof

$$
\begin{aligned}
\dot{V}(x) & =\frac{1}{2}\left(\dot{x}^{T} Q x+x^{T} Q \dot{x}\right)=\frac{1}{2} x^{T}\left(A^{T} Q+Q A\right) x, \quad \text { and thus } \\
\dot{V}(\xi) & =\frac{1}{2} \xi^{T}\left(A^{T} Q+Q A\right) \xi=\frac{1}{2} 2 \lambda \xi^{T} Q \xi=2 \lambda V(\xi) .
\end{aligned}
$$

Thus

$$
\lambda=\frac{\dot{V}(\xi)}{V(\xi)}
$$

Now

$$
\begin{aligned}
\frac{\dot{V}\left(x_{r}\right)}{V\left(x_{r}\right)} & =\frac{x_{r}^{T}\left(A^{T} Q+Q A\right) x_{r}}{x_{r}^{T} Q x_{r}} \\
& =\frac{\left(\lambda_{r} x_{r}^{T}-\lambda_{i} x_{i}^{T}\right) Q x_{r}+x_{r}^{T} Q\left(\lambda_{r} x_{r}-\lambda_{i} x_{i}\right)}{x_{r}^{T} Q x_{r}} \\
& =2 \lambda_{r}-2 \lambda_{i} \frac{x_{i}^{T} Q x_{r}}{x_{r}^{T} Q x_{r}} .
\end{aligned}
$$

Similarly,

$$
\frac{\dot{V}\left(x_{i}\right)}{V\left(x_{i}\right)}=2 \lambda_{r}+2 \lambda_{i} \frac{x_{i}^{T} Q x_{r}}{x_{i}^{T} Q x_{i}} .
$$

Adding suitable multiples of (5.2) and (5.3) we get,

$$
\begin{aligned}
2 \lambda_{r}\left(x_{r}^{T} Q x_{r}+x_{i}^{T} Q x_{i}\right) & =\frac{\dot{V}\left(x_{r}\right)}{V\left(x_{r}\right)} x_{r}^{T} Q x_{r}+\frac{\dot{V}\left(x_{i}\right)}{V\left(x_{i}\right)} x_{i}^{T} Q x_{i} \\
& =2\left(\dot{V}\left(x_{r}\right)+\dot{V}\left(x_{i}\right)\right)
\end{aligned}
$$

Therefore,

$$
\lambda_{r}=\frac{\dot{V}\left(x_{r}\right)+\dot{V}\left(x_{i}\right)}{x_{r}^{T} Q x_{r}+x_{i}^{T} Q x_{i}}=\frac{\dot{V}\left(x_{r}\right)+\dot{V}\left(x_{i}\right)}{2\left(V\left(x_{r}\right)+V\left(x_{i}\right)\right)} .
$$

Corollary 5.2 Consider the matrix Lyapunov equation

$$
A^{T} X+X A=-P
$$

associated to the linear system $\dot{x}=A x$, where $P=P^{T}>0$ is given. Suppose $Q=Q^{T}$ is a solution to (5.4). Then,

$$
\operatorname{card}\left\{\lambda \mid \lambda \in \operatorname{spectrum}(A), \operatorname{Re}(\lambda)=\lambda_{r}>0\right\} \leq \operatorname{index}(Q),
$$

where $\operatorname{index}(Q)$ means the number of negative eigenvalues of $Q$. 
Proof In Lemma 5.1, choose $Q$ to be a solution to the Lyapunov equation (5.4). Then,

$$
\begin{aligned}
\dot{V}(x) & =\frac{1}{2}\left(\dot{x}^{T} Q x+x^{T} Q \dot{x}\right) \\
& =\frac{1}{2} x^{T}\left(A^{T} Q+Q A\right) x \\
& =-\frac{1}{2} x^{T} P x
\end{aligned}
$$

From Lemma 5.1, for any eigenvalue $\lambda$ of $A$,

$$
\begin{aligned}
\lambda_{r} & =\frac{\dot{V}\left(x_{r}\right)+\dot{V}\left(x_{i}\right)}{2\left(V\left(x_{r}\right)+V\left(x_{i}\right)\right.} \\
& =\frac{-\frac{1}{2}\left(x_{r}^{T} P x_{r}+x_{i}^{T} P x_{i}\right)}{\left(x_{r}^{T} Q x_{r}+x_{i}^{T} Q x_{i}\right)} \\
& =-\frac{1}{2} \frac{\bar{\xi}^{T} P \xi}{\bar{\xi}^{T} Q \xi}
\end{aligned}
$$

Since $P=P^{T}>0, \lambda_{r}>0$, this implies $\bar{\xi}^{T} Q \xi<0$.

Remark It is well-known that when $\operatorname{spectrum}(A)$ lies in the strict left half plane, (5.4) has the unique positive definite solution

$$
\int_{0}^{\infty} e^{A^{T} \sigma} Q e^{A \sigma} d \sigma
$$

If in Corollary 5.2, we impose the additional condition that, for any $\lambda, \mu \in \operatorname{spectrum}(A), \lambda+$ $\mu \neq 0$, then, the inequality (5.5) becomes an equality. This is a theorem of Taussky [1961].

Suppose the linear system of interest is

$$
\dot{x}=\left[\Omega^{-1}\right]^{T} Q x+\epsilon B x
$$

where $\Omega=-\Omega^{T}$ is a nonsingular matrix (e.g. the symplectic structure) of size $2 n \times$ $2 n$, the matrix $B$ is symmetric and determines a, possibly dissipative, perturbation, $\epsilon \geq 0$ is a small parameter, and $Q=Q^{T}$ determines the energy quadratic form

$$
E(x)=\frac{1}{2} x^{T} Q x
$$

for the underlying unperturbed system. Along trajectories of (5.6)

$$
\dot{E}(x)=\epsilon x^{T}(Q B+B Q) x .
$$


Corollary 5.3 Suppose $\lambda$ is a simple eigenvalue of $A=\Omega^{-T} Q$ with eigenvector $\xi=$ $x_{r}+i x_{i}$. Let $\lambda_{r}^{\epsilon}$ denote the real part of the eigenvalue branch $\lambda^{\epsilon}$ of $A_{\epsilon}=\Omega^{-T} Q+\epsilon B$ emanating from $\lambda$. Then

$$
\begin{aligned}
\lambda_{r}^{\prime}: & =\left.\frac{d}{d \epsilon} \lambda_{r}^{\epsilon}\right|_{\epsilon=0} \\
& =\frac{1}{2} \frac{\dot{E}^{\prime}\left(x_{r}\right)+\dot{E}^{\prime}\left(x_{i}\right)}{E\left(x_{r}\right)+E\left(x_{i}\right)}
\end{aligned}
$$

where

$$
\dot{E}^{\prime}(x)=x^{T}(Q B+B Q) x .
$$

Proof Substitute $E(x)$ for $V(x)$ in Lemma $\mathbf{5 . 1}$ and observe that simplicity of $\lambda$ ensures smoothness of $\dot{E}\left(x_{r}^{\epsilon}\right), \dot{E}\left(x_{i}^{\epsilon}\right)$ etc. with respect to $\epsilon$ at $\epsilon=0$.

If in Corollary 5.3, the eigenvalue branch $\lambda^{\epsilon}$ is emanating from a pure imaginary eigenvalue $\lambda=i \omega$, then the formula (5.9) becomes a formula for the crossing speed. It is our aim to make this formula explicit for systems in block-diagonal normal form. As a first step we note

Lemma 5.4 Under the hypotheses of Corollary 5.3, and if $\lambda=i \omega$ where $\omega \in \mathbb{R}$,

$$
\lambda_{r}^{\prime}=\frac{\bar{\xi}^{T}(\Omega B)_{\text {anti }} \xi}{\bar{\xi}^{T} \Omega \xi},
$$

where $(\cdot)$ anti denotes the anti-symmetric part.

Proof Since $A\left(x_{r}+i x_{i}\right)=i \omega\left(x_{r}+i x_{i}\right)$, we have

$$
A x_{r}=-\omega x_{i}=\frac{i \omega}{2}(\xi-\bar{\xi}),
$$

and

$$
A x_{i}=\omega x_{r}=\frac{\omega}{2}(\xi+\bar{\xi})
$$

Then,

$$
\begin{aligned}
E\left(x_{r}\right) & =\frac{1}{2} x_{r}^{T} Q x_{r}=\frac{1}{2} x_{r}^{T} \Omega^{T} A x_{r} \\
& =\frac{1}{2}\left(\frac{\xi+\bar{\xi}}{2}\right)^{T} \Omega^{T} \frac{i \omega}{2}(\xi-\bar{\xi}) \\
& =-\frac{1}{4} i \omega \bar{\xi}^{T} \Omega \xi
\end{aligned}
$$

By antisymmetry of $\Omega,-\xi^{T} \Omega \bar{\xi}=\bar{\xi}^{T} \Omega \xi$ and hence

$$
\begin{aligned}
E\left(x_{i}\right) & =\frac{1}{2} x_{i}^{T} Q x_{i}=\frac{1}{2} x_{i}^{T} \Omega^{T} A x_{i} \\
& =\frac{1}{2}\left(\frac{\xi-\bar{\xi}}{2 i}\right)^{T} \Omega^{T} \frac{\omega}{2}(\xi+\bar{\xi}) \\
& =-\frac{1}{4} i \omega \bar{\xi}^{T} \Omega \xi
\end{aligned}
$$


Further, since $Q=-\Omega A=A^{T} \Omega$, formula (88) implies

$$
\begin{aligned}
\dot{E}^{\prime}\left(x_{r}\right)+\dot{E}^{\prime}\left(x_{i}\right)= & \frac{1}{2} x_{r}^{T}(Q B+B Q) x_{r}+x_{i}^{T}(Q B+B Q) x_{i} \\
= & \frac{1}{2} x_{r}^{T} A^{T} \Omega B x_{r}-\frac{1}{2} x_{r}^{T} B \Omega A x_{r}+\frac{1}{2} x_{i}^{T} A^{T} \Omega B x_{i}-\frac{1}{2} x_{i}^{T} B \Omega A x_{i} \\
= & \frac{i \omega}{8}(\xi-\bar{\xi})^{T} \Omega B(\xi+\bar{\xi})-\frac{i \omega}{8}(\xi+\bar{\xi})^{T} B \Omega(\xi-\bar{\xi}) \\
& -\frac{i \omega}{8}(\xi+\bar{\xi})^{T} \Omega B(\xi-\bar{\xi})+\frac{i \omega}{8}(\xi-\bar{\xi})^{T} B \Omega(\xi+\bar{\xi}) \\
= & \frac{i \omega}{4}\left(\xi^{T} \Omega B \bar{\xi}-\bar{\xi}^{T} \Omega B \xi\right)+\frac{i \omega}{4}\left(\xi^{T} B \Omega \bar{\xi}-\bar{\xi}^{T} B \Omega \xi\right) \\
= & \frac{i \omega}{2} \xi^{T} \frac{\Omega B-(\Omega B)^{T}}{2} \bar{\xi}-\frac{i \omega}{2} \bar{\xi}^{T} \frac{\Omega B-(\Omega B)^{T}}{2} \xi \\
= & -\frac{i \omega}{2}\left(-\xi^{T}(\Omega B)_{\mathrm{anti}} \bar{\xi}+\bar{\xi}^{T}(\Omega B)_{\mathrm{anti}} \xi\right) \\
= & -i \omega \bar{\xi}^{T}(\Omega B)_{\mathrm{anti}} \xi
\end{aligned}
$$

From (5.12), (5.13) and (5.14) we get,

$$
\lambda_{r}^{\prime}=\frac{\dot{E}^{\prime}\left(x_{r}\right)+\dot{E}^{\prime}\left(x_{i}\right)}{2\left(E\left(x_{r}\right)+E\left(x_{i}\right)\right)}=\frac{\bar{\xi}^{T}(\Omega B)_{\mathrm{anti}} \xi}{\bar{\xi}^{T} \Omega \xi} .
$$

Remark The special case $\Omega=J=\left[\begin{array}{cc}0 & I \\ -I & 0\end{array}\right]$ of formula (5.11) appears in R. McKay [1991] who also gives it an averaging interpretation. We note that our result is a corollary of the more general formula (5.9) which applies to eigenvalues that are not necessarily on the imaginary axis. The proof presented here does not use the Kato perturbation lemma involving both right and left eigenvectors - the key tool in McKay's argument.

Next we compute the average $\left\langle\dot{E}^{\prime}\left(x_{r}\right)\right\rangle$ over a cycle of period $2 \pi / \omega$ of the periodic solution $\xi e^{i \omega t}$ for the unperturbed system. Recall that at $t=0$, the formula

$$
\dot{E}^{\prime}\left(x_{r}\right)=\frac{i \omega}{4}(\xi-\bar{\xi})^{T} \Omega B(\xi+\bar{\xi})
$$

holds. For any other $t$,

$$
\begin{aligned}
\dot{E}^{\prime}\left(x_{r}(t)\right) & =\frac{i \omega}{4}\left(\xi e^{i \omega t}-\bar{\xi} e^{-i \omega t}\right)^{T} \Omega B\left(\xi e^{i \omega t}+\bar{\xi} e^{-i \omega t}\right) \\
& =\frac{i \omega}{4}\left\{\xi^{T} \Omega B e^{i 2 \omega t}-\bar{\xi}^{T} \Omega B \xi+\xi^{T} \Omega B \bar{\xi}-\bar{\xi}^{T} \Omega B \xi e^{-2 i \omega t}\right\}
\end{aligned}
$$

Substituting from (5.15) into the average defined by

$$
\left\langle\dot{E}^{\prime}\left(x_{r}\right)\right\rangle:=\frac{1}{2 \pi / \omega} \int_{0}^{2 \pi / \omega} \dot{E}^{\prime}\left(x_{r}(t)\right) d t
$$


we get,

$$
\left\langle\dot{E}^{\prime}\left(x_{r}\right)\right\rangle=\frac{i \omega}{4}\left(\xi^{T} \Omega B \bar{\xi}-\bar{\xi}^{T} \Omega B \xi\right)=-\frac{i \omega}{4}\left(\bar{\xi}^{T}(\Omega B)_{\mathrm{anti}} \xi\right) .
$$

In evaluating (5.16) we used the fact that $\int_{0}^{2 \pi / \omega} e^{i k \omega t} d t=0$ for any nonzero integer $k$. From (5.14) and (5.11), and (5.12), (5.13) we get,

$$
\lambda_{r}^{\prime}=\frac{\left\langle\dot{E}^{\prime}\left(x_{r}\right)\right\rangle}{2 E\left(x_{r}\right)} .
$$

This is the averaging interpretation of the crossing speed given by McKay in the case $\Omega=J$.

In the remainder of this section we show how to adapt the crossing-speed result (5.11) to the block-diagonal normal form. Recall that the symplectic structure of the block-diagonal normal form is not canonical. It is of the form "coadjoint orbit, internal symplectic, magnetic and coupling terms";

$$
\Omega=\left[\begin{array}{ccc}
L_{\mu} & C & 0 \\
-C^{T} & S & \mathbf{1} \\
0 & -\mathbf{1} & 0
\end{array}\right]
$$

The second variation $\delta^{2} H_{\xi}$ takes the form,

$$
Q=\left[\begin{array}{ccc}
A_{\mu} & 0 & 0 \\
0 & \Lambda & 0 \\
0 & 0 & M^{-1}
\end{array}\right]
$$

and the dissipatively perturbed linear system of interest is (cf. equation (3.2))

$$
\dot{x}=(A+\epsilon B) x=\left(\Omega^{-T} Q+\epsilon B\right) x
$$

where

$$
B=\left[\begin{array}{ccc}
0 & 0 & 0 \\
0 & 0 & 0 \\
0 & 0 & -R M^{-1}
\end{array}\right] .
$$

Here $x=(r, q, p)$, as in $\S$ 3. A key stumbling block in using the crossing speed formula (5.11) is the need to calculate the eigenvector $\xi$ corresponding to the eigenvalue $i \omega$. The following result eases the way a little.

Lemma 5.5 Consider the quadratic pencil

$$
G(\lambda)=\left[\begin{array}{cc}
\lambda^{2} M+\lambda S+\Lambda & -\lambda C^{T} \\
-\lambda L_{\mu}^{-T} C & \lambda \mathbf{1}-L_{\mu}^{-T} C
\end{array}\right] .
$$

Then $\lambda_{0}$ is a singular point of the pencil, i.e., det $\left[G\left(\lambda_{0}\right)\right]=0$, with corresponding null-vector $y_{0}=\left(q_{0}^{T}, r_{0}^{T}\right)^{T}$ iff $\lambda_{0}$ is an eigenvalue of $A$ with eigenvector $\xi=$ $\left(r_{0}^{T}, q_{0}^{T}, \lambda_{0}\left(M q_{0}\right)^{T}\right)^{T}$. 
Proof Note the equivalence between the unperturbed system $\dot{x}=A x$ and the coupled system consisting of the second-order internal dynamics together with the first order coadjoint orbit dynamics, in normal form:

$$
\begin{aligned}
M \ddot{q}+S \dot{q}+\Lambda q & =C^{T} \dot{r} \\
\dot{r} & =L_{\mu}^{-T} A_{\mu} r+L_{\mu}^{-T} C \dot{q} .
\end{aligned}
$$

Interpret $G(\lambda)$ as the Laplace transform representation of (5.24). This immediately identifies singular points of the pencil $G(\lambda)$ with the spectrum of $A$. The eigenvectornull vector result is a direct calculation.

Now, suppose $\lambda=i \omega_{0}$ is a pure imaginary eigenvalue of $A$ (singular point of $G(\lambda))$. Let

$$
G\left(i \omega_{0}\right)\left(\begin{array}{c}
q_{0} \\
r_{0}
\end{array}\right)=0
$$

By Lemma 5.5, and verifying that

$$
(\Omega B)_{\mathrm{anti}}=\frac{1}{2}\left[\begin{array}{ccc}
0 & 0 & 0 \\
0 & 0 & -R M^{-1} \\
0 & R M^{-1} & 0
\end{array}\right],
$$

we get

$$
\bar{\xi}^{T}(\Omega B)_{\mathrm{anti}} \xi=-i \omega_{0} \bar{q}_{0}^{T} R q_{0} .
$$

Again by Lemma $\mathbf{5 . 5}$ and (5.19),

$$
\bar{\xi}^{T} \Omega \xi=\bar{r}_{0}^{T} L_{\mu} r_{0}+2 i \omega_{0} \bar{q}_{0}^{T} M \bar{q}_{0}+\bar{q}_{0}^{T} S q_{0}+\bar{r}_{0}^{T} C q_{0}-\bar{q}_{0}^{T} C r_{0} .
$$

Setting,

$$
\begin{aligned}
& q_{0}=\eta+i \beta, \\
& r_{0}=u+i v
\end{aligned}
$$

and substituting in (5.25), (5.26) we get the following "block-diagonal" version of the crossing speed formula,

$$
\lambda_{r}^{\prime}=\frac{-\omega_{0}\left(\eta^{T} R \eta+\beta^{T} R \beta\right)}{2\left\{u^{T} L_{\mu} v+u^{T} C \beta-v^{T} C \eta+\eta^{T} S \beta+\omega_{0}\left(\eta^{T} M \eta+\beta^{T} M \beta\right)\right\}} .
$$

Remark The crossing speed formula (5.27) can lead to effective computation provided one has some insight into $\operatorname{det}\left(G\left(i \omega_{0}\right)\right)$ and can compute a null vector of $G\left(i \omega_{0}\right)$. This is still more manageable than directly computing eigenvectors of $A$ due to the smaller matrices involved. 
Remark In the abelian case, $L_{\mu}=0=C$ and the crossing speed formula (5.27) specializes to

$$
\lambda_{r}^{\prime}=\frac{-\omega_{0}\left(\eta^{T} R \eta+\beta^{T} R \beta\right)}{2\left\{\omega_{0}\left(\eta^{T} M \eta+\beta^{T} M \beta\right)+\eta^{T} S \beta\right\}} .
$$

For a similar formula for two degree of freedom systems, see Haller [1992]. Further, if there is no gyroscopic/magnetic term, i.e., $S=0$ then (5.28) predicts that every pure imaginary eigenvalue of the unperturbed system is pushed into the left half plane under a strong dissipation $R>0$. Of course, this says nothing about any eigenvalues of the unperturbed system that may be in the right half plane - such eigenvalues are bound to be present if $S=0$ and $\Lambda$ is indefinite.

Example As we already saw in the introductory section, for the two degrees of freedom Chetaev problem ( $c f$. equation (1.9)),

$$
\begin{aligned}
\ddot{x}-g \dot{y}+\epsilon \gamma \dot{x}+\alpha x & =0 \\
\ddot{y}+g \dot{x}+\epsilon \delta \dot{y}+\beta y & =0,
\end{aligned}
$$

if $\alpha$ and $\beta$ are both negative, and if we set $\epsilon=0$, then for $g^{2}+\alpha+\beta>2 \sqrt{\alpha \beta}$, all eigenvalues are pure imaginary (the unperturbed system is gyroscopically stable). But, for strong dissipation, $\gamma>0, \delta>0$ and $\epsilon>0$, one pair of eigenvalues crosses into the right half plane and another pair into the left half plane. This was shown by a Routh-Hurwitz calculation which, being a counting device, is not capable of telling us which eigenvalue crosses over to which half plane. Employing the crossing speed formula (5.28) we are able to address precisely this problem of tracking eigenvalue movement.

Note that the Chetaev problem is in the abelian case with

$$
\begin{aligned}
M & =\left[\begin{array}{ll}
1 & 0 \\
0 & 1
\end{array}\right] ; S=\left[\begin{array}{cc}
0 & -g \\
g & 0
\end{array}\right] ; R=\left[\begin{array}{ll}
\gamma & 0 \\
0 & \delta
\end{array}\right] \\
\Lambda & =\left[\begin{array}{ll}
\alpha & 0 \\
0 & \beta
\end{array}\right] .
\end{aligned}
$$

One checks that

$$
\operatorname{det}\left(G\left(i \omega_{0}\right)\right)=\left(-\omega_{0}^{2}+\alpha\right)\left(-\omega_{0}^{2}+\beta\right)-\omega_{0}^{2} g^{2}=0,
$$

which determines two distinct pairs of pure imaginary eigenvalues if $g^{2}+\alpha+\beta>$ $2 \sqrt{\alpha \beta}$. Corresponding to $\lambda=i \omega_{0}$, a null-vector for $G\left(i \omega_{0}\right)$ is given by,

$$
\left[\begin{array}{l}
q_{1} \\
q_{2}
\end{array}\right]=\left[\begin{array}{c}
i g \omega_{0} \\
-\omega_{0}^{2}+\alpha
\end{array}\right]=\eta+i \beta
$$

Thus $\eta=\left(0,-\omega_{0}^{2}+\alpha\right)^{T}, \beta=\left(g \omega_{0}, 0\right)^{T}$. Substituting in (5.28) we get,

$$
\lambda_{r}^{\prime}=\frac{-\frac{1}{2}\left\{\delta\left(-\omega_{0}^{2}+\alpha\right)^{2}+\gamma g^{2} \omega_{0}^{2}\right\}}{\left\{\left(-\omega_{0}^{2}+\alpha\right)^{2}+g^{2} \omega_{0}^{2}+g^{2}\left(-\omega_{0}^{2}+\alpha\right)\right\}} .
$$




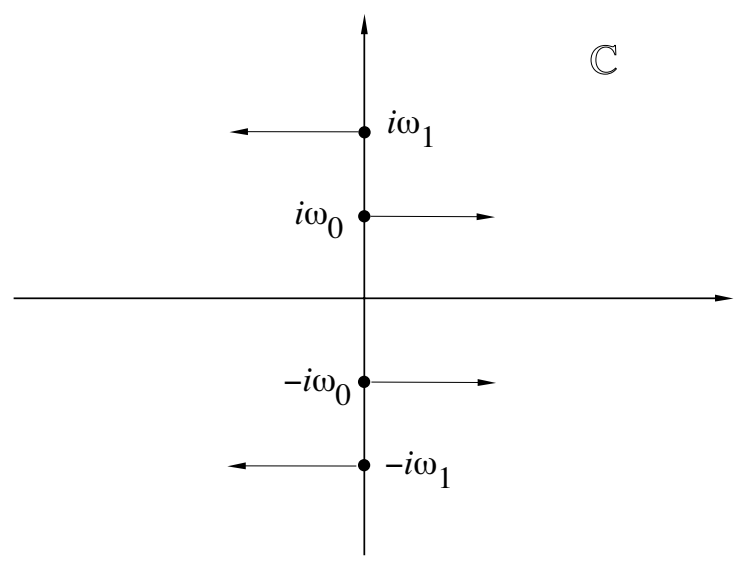

Figure 3: The weaker get destabilized.

Using the relation (5.31) we can simplify further to obtain

$$
\lambda_{r}^{\prime}=-\frac{1}{2} \frac{\left\{\delta\left(-\omega_{0}^{2}+\alpha\right)+\gamma\left(-\omega_{0}^{2}+\beta\right)\right\}}{\left\{\left(-\omega_{0}^{2}+\alpha\right)+\left(-\omega_{0}^{2}+\beta\right) g^{2}\right\}} .
$$

Suppose $\pm i \omega_{0}$ and $\pm i \omega_{1}$ are the distinct eigenvalues of the unperturbed system. Then,

$$
\omega_{0}^{2}+\omega_{1}^{2}=g^{2}+\alpha+\beta
$$

Therefore,

$$
\left(-\omega_{0}^{2}+\alpha\right)+\left(-\omega_{0}^{2}+\beta\right)+g^{2}=\omega_{1}^{2}+\omega_{0}^{2}-\omega_{0}^{2}-\omega_{0}^{2}=\omega_{1}^{2}-\omega_{0}^{2} .
$$

Thus,

$$
\lambda_{r}^{\prime}=\frac{-\frac{1}{2}\left\{\delta\left(-\omega_{0}^{2}+\alpha\right)+\gamma\left(-\omega_{0}^{2}+\beta\right)\right\}}{\left\{\omega_{1}^{2}-\omega_{0}^{2}\right\}} .
$$

By hypothesis, $\alpha<0, \beta<0, \delta>0, \gamma>0$. It follows that

$$
-\frac{1}{2}\left\{\delta\left(-\omega_{0}^{2}+\alpha\right)+\gamma\left(-\omega_{0}^{2}+\beta\right)\right\}>0 .
$$

Hence the simple eigenvalue $\pm i \omega_{0}$ moves to the right (left) half plane according as whether $\omega_{1}>\omega_{0}\left(\omega_{1}<\omega_{0}\right)$. See Figure 5.1.

Example The simplest non-abelian case arises when $G=S O(3)$ and the shape space dimension is 1. A physical example of this is that of a rigid body with an attached pointmass at the end of a spring, free to oscillate along a linear guideway. First, note that we can do some basic calculations without reference to a particular equilibrium about which block-diagonal normal form is used. Let,

$$
L_{\mu}=\left[\begin{array}{cc}
0 & -g \\
g & 0
\end{array}\right] ; C=\left[\begin{array}{l}
C_{1} \\
C_{2}
\end{array}\right] ; A_{\mu}=\left[\begin{array}{ll}
a_{11} & a_{12} \\
a_{12} & a_{22}
\end{array}\right]>0 .
$$


Note that $C^{T}$ is not injective, a case not covered by Theorem 3.3. The magnetic term $S=-S^{T}=0$, since the shape space dimension is 1 by hypothesis. Let $\Lambda=\alpha$ and $M=m$ be the scalar stiffness and mass respectively. The quadratic pencil of Lemma $\mathbf{5 . 5}$ takes the form

$$
G(\lambda)=\left[\begin{array}{ccc}
\lambda^{2} m+\alpha & -\lambda C_{1} & -\lambda C_{2} \\
-\lambda C_{2} / g & \lambda-\frac{a_{12}}{g} & -\frac{a_{22}}{g} \\
\lambda C_{1} / g & \frac{a_{11}}{g} & \lambda+\frac{a_{12}}{g}
\end{array}\right] .
$$

It can be verified that

$$
p(\lambda)=\operatorname{det} G(\lambda)=m \lambda^{4}+\lambda^{2}\left\{\alpha+\frac{m}{g^{2}} \Delta_{1}+\frac{\Delta_{2}}{g^{2}}\right\}+\alpha \frac{\Delta_{1}}{g^{2}},
$$

where

$$
\Delta_{1}=\frac{a_{11} a_{22}-a_{12}^{2}}{g^{2}}>0
$$

(because $A_{\mu}>0$ ) and

$$
\begin{aligned}
\Delta_{2} & =\frac{C_{1}^{2} a_{22}+C_{2}^{2} a_{11}-2 C_{1} C_{2} a_{12}}{g^{2}} \\
& =\frac{1}{g^{2}}\left[C_{2}-C_{1}\right]\left[\begin{array}{ll}
a_{11} & a_{12} \\
a_{12} & a_{22}
\end{array}\right]\left[\begin{array}{c}
C_{2} \\
-C_{1}
\end{array}\right]>0
\end{aligned}
$$

(again because $A_{\mu}>0$ ).

There are three cases to consider:

(a) If $\alpha>0$, then the second variation is postive definite and all the roots of $p(\lambda)$, (i.e., eigenvalues of the unperturbed Hamiltonian system) are pure imaginary.

(b) If $\alpha=0$, then there is repeated root at the origin and a pure imaginary pair $\pm i \omega_{0}$.

(c) If $\alpha<0$, two of the roots of $p(\lambda)$ are real with one root lying in the right half plane.

In case (a), a dissipative perturbation moves the eigenvalues into the left half plane. This is already covered by the general theory, but can be recovered by the crossing-speed formula (5.27) with $S=0$, a calculation left to the reader. Case (c) is the odd-index case and the Cartan-Chetaev-Oh lemma demonstrates instability with or without added dissipation. In case (b) our crossing speed formula (5.27) applies to the pair of pure imaginary roots (since they are simple). The details are again left to the reader. 


\section{$6 \quad$ Examples}

Example 1 (The Rigid Body with Internal Rotors) Consider a rigid body with two symmetric rotors. It is assumed that the rotors are subject to a dissipative/frictional torque and no other forcing. A steady spin about the minor axis of the locked inertia tensor ellipsoid (i.e., the long axis of the body), is a relative equilibrium. Without friction, this system can experience gyroscopic stabilization and the second variation of the augmented Hamiltonian can be indefinite. We aim to show that this is an unstable relative equilibrium with dissipation added.

The equations of motion are (see Krishnaprasad [1985] and Bloch, Krishnaprasad, Marsden, and Sanchez de Alvarez [1992]):

$$
\begin{aligned}
& \left(\mathbb{I}_{\text {lock }}-\mathbb{I}_{\text {rotor }}\right) \dot{\Omega}=\left(\mathbb{I}_{\text {lock }} \Omega+\mathbb{I}_{\text {rotor }} \Omega_{r}\right) \times \Omega \\
& \dot{\Omega}_{r}=-\left(\mathbb{I}_{\text {lock }}-\mathbb{I}_{\text {rotor }}\right)^{-1}\left(\mathbb{I}_{\text {lock }} \Omega+\mathbb{I}_{\text {rotor }} \Omega_{r}\right) \times \Omega-R \Omega_{r} \\
& \dot{A}=A \hat{\Omega} \\
& \dot{\theta}_{r}=\Omega_{r}
\end{aligned}
$$

In this example, $Q=S O(3) \times S^{1} \times S^{1}$ and $G=S O(3)$. Also $A \in S O(3)$ denotes the attitude/orientation of the carrier rigid body relative to an inertial frame, $\Omega \in \mathbb{R}^{3}$ is the body angular velocity of the carrier, $\Omega_{r} \in \mathbb{R}^{3}$ is the vector of angular velocities of the rotors in the body frame (with third component set equal to zero) and $\theta_{r}$ is the ordered set of rotor angles in body frame (again, with third component set equal to zero). Further, $\mathbb{I}_{\text {lock }}$ denotes the moment of inertia of the body and locked rotors in the body frame and $\mathbb{I}_{\text {rotor }}$ is the $3 \times 3$ diagonal matrix of rotor inertias. We let

$$
\left.\begin{array}{rl}
\mathbb{I}_{\text {lock }} & =\operatorname{diag}\left(B_{1}, B_{2}, B_{3}\right), \\
\mathbb{I}_{\text {rotor }} & =\operatorname{diag}\left(J_{1}^{1}, J_{2}^{2}, 0\right), \\
\mathbb{I}_{\text {lock }}-\mathbb{I}_{\text {rotor }} & =\operatorname{diag}\left(A_{1}, A_{2}, A_{3}\right) .
\end{array}\right\}
$$

Assume that $B_{1}>B_{2}>B_{3}$. Finally, $R=\operatorname{diag}\left(R_{1}, R_{2}, 0\right)$ is the matrix of rotor dissipation coefficients, $R_{i}>0$.

Consider the relative equilibrium for (113) defined by, $\Omega^{e}=(0,0, \omega)^{T} ; \Omega_{r}^{e}=$ $(0,0,0)^{T}$ and $\theta_{r}=\theta_{r}^{e}$ an arbitrary constant. This corresponds to a steady minor axis spin of the rigid body with the two rotors non-spinning. Linearization of the $S O(3)$-reduction of (113) about this equilibrium yields,

$$
\begin{aligned}
\left(\mathbb{I}_{\text {lock }}-\right. & \left.\mathbb{I}_{\text {rotor }}\right) \delta \dot{\Omega}=\left(\mathbb{I}_{\text {lock }} \delta \Omega+\mathbb{I}_{\text {rotor }} \delta \Omega_{r}\right) \times \Omega^{e}+\left(\mathbb{I}_{\text {lock }} \Omega^{e}\right) \times \delta \Omega \\
\delta \dot{\Omega}_{r}= & -\left(\mathbb{I}_{\text {lock }}-\mathbb{I}_{\text {rotor }}\right)^{-1}\left[\left(\mathbb{I}_{\text {lock }} \delta \Omega+\mathbb{I}_{\text {rotor }} \delta \Omega_{r}\right) \times \Omega^{e}\right. \\
& \left.+\left(\mathbb{I}_{\text {lock }} \Omega^{e}\right) \times \delta \Omega\right]-R \delta \Omega_{r} \\
\delta \dot{\theta}_{r}= & \delta \Omega_{r}
\end{aligned}
$$


It is easy to verify that $\delta \dot{\Omega}_{3}=0$. This reflects the choice of relative equilibrium. Similarly $\delta \dot{\Omega}_{r_{3}}=0$. We will now apply Theorem $\mathbf{3 . 3}$ in the case of $\Lambda=0$.

Dropping the kinematic equations for $\delta \theta_{r}$ we have the "reduced" linearized equations

$$
\left[\begin{array}{c}
\delta \dot{\Omega}_{r_{1}} \\
\delta \dot{\Omega}_{r_{2}} \\
\delta \dot{\Omega}_{1} \\
\delta \dot{\Omega}_{2}
\end{array}\right]=\left[\begin{array}{cccc}
-R_{1} & \frac{-J_{2}^{2} \omega}{A_{1}} & 0 & \frac{B_{3}-B_{2}}{A_{1}} \omega \\
\frac{J_{1}^{1} \omega}{A_{2}} & -R_{2} & \frac{B_{1}-B_{3}}{A_{2}} \omega & 0 \\
0 & \frac{J_{2}^{2} \omega}{A_{1}} & 0 & \frac{B_{2}-B_{3}}{A_{1}} \omega \\
\frac{-J_{1}^{1} \omega}{A_{2}} & 0 & \frac{B_{3}-B_{1}}{A_{2}} \omega & 0
\end{array}\right]\left[\begin{array}{c}
\delta \Omega_{r_{1}} \\
\delta \Omega_{r_{2}} \\
\delta \Omega_{1} \\
\delta \Omega_{2}
\end{array}\right] .
$$

Assume that $\omega \neq 0$ (nondegeneracy of the relative equilibrium). Then the above equations are easily verified to be in the normal form (3.18), upon making the identifications, $p=\left(\delta \Omega_{r_{1}}, \delta \Omega_{r_{2}}\right)^{T}, q=\left(\delta \Omega_{1}, \delta \Omega_{2}\right)^{T}$, and,

$$
\begin{aligned}
L_{\mu} & =\left[\begin{array}{cc}
0 & -1 / \omega \\
1 / \omega & 0
\end{array}\right] ; C=\left[\begin{array}{cc}
-1 & 0 \\
0 & -1
\end{array}\right] ; \tilde{S}=\left[\begin{array}{cc}
0 & \omega \\
-\omega & 0
\end{array}\right] \\
A_{\mu} & =\left[\begin{array}{cc}
\frac{B_{3}-B_{1}}{A_{2}} & 0 \\
0 & \frac{B_{3}-B_{2}}{A_{1}}
\end{array}\right] ; M^{-1}=\left[\begin{array}{cc}
\frac{J_{1}^{1}}{A_{2}} & 0 \\
0 & \frac{J_{2}^{2}}{A_{1}}
\end{array}\right] ; \\
R & =\left[\begin{array}{cc}
R_{1} \frac{A_{2}}{J_{1}^{1}} & 0 \\
0 & R_{2} \frac{A_{1}}{J_{2}^{2}}
\end{array}\right] .
\end{aligned}
$$

Since $B_{1}>B_{2}>B_{3}, A_{\mu}$ is negative definite. Also, $M$ and $R$ are positive definite, and $C^{T}$ is injective and thus all the hypotheses of Theorem $\mathbf{3 . 3}$ are satisfied. Thus the linearized system (6) or (115) displays dissipation-induced instability.

Remark In the body and rotors example, the linearized system was shown to be in block-diagonal normal form by inspection. Our calculations also reveal that there is some freedom in the choice of block-diagonal parameters-for instance the scalar $\omega$ could appear in various ways in $L_{\mu}, C$ etc.

Remark This example is also instructive in that we can verify the instability result by a Routh-Hurwitz computation, as in Proposition 1.2. We sketch the computation here and note that calculations like this can sometimes be tedious, indicating the usefulness of the general result, even in this relatively low dimensional case.

A straightforward calculation yields the following characteristic polynomial of 
the linearized system (6.3) or (6.4), with no dissipation,

$$
p(\lambda)=\lambda^{4}+\omega^{2} \lambda^{2} \frac{\left(J_{2}^{2}-\left(B_{2}-B_{3}\right)\right)\left(J_{1}^{1}-\left(B_{1}-B_{3}\right)\right)}{A_{1} A_{2}} .
$$

There are two eigenvalues at the origin, consistent with the rank deficit of 2 in $\left[\begin{array}{cc}L_{\mu}^{-T} & L_{\mu}^{-T} C \\ C^{T} L_{\mu}^{-T} & -\tilde{S}\end{array}\right]$ and, under the additional physical assumption that

$$
\left(J_{2}^{2}-\left(B_{2}-B_{3}\right)\right)\left(J_{1}^{1}-\left(B_{1}-B_{3}\right)\right)>0,
$$

the other two eigenvalues are pure imaginary. In fact, we assume both factors in the preceding equation (6.6) are negative, since the rotor inertias are small. Now consider the case in which $R_{1}, R_{2}>0$, and are small. The full characteristic polynomial is

$$
\begin{aligned}
\lambda^{4}+ & \lambda^{3}\left(R_{1}+R_{2}\right) \\
& +\omega^{2} \lambda^{2}\left\{\frac{R_{1} R_{2}}{\omega^{2}}+\frac{\left(J_{2}^{2}-\left(B_{2}-B_{3}\right)\right)\left(J_{1}^{1}-\left(B_{1}-B_{2}\right)\right)}{A_{1} A_{2}}\right\} \\
& -\omega^{2} \frac{\lambda}{A_{1} A_{2}}\left\{R_{1}\left(B_{1}-B_{3}\right)\left(J_{2}^{2}-\left(B_{2}-B_{3}\right)\right)+R_{2}\left(B_{2}-B_{3}\right)\left(J_{1}^{1}-\left(B_{1}-B_{3}\right)\right)\right\} \\
& +\omega^{2}\left\{\frac{\left(B_{2}-B_{3}\right)\left(B_{1}-B_{3}\right) R_{1} R_{2}}{A_{1} A_{2}}\right\} .
\end{aligned}
$$

Now, use the same notation for the characteristic polynomial (6.7) as in Proposition 1.2. We need to compute the sign changes in the sequence (1.14). Clearly $\rho_{1}$ and $\rho_{4}$ are positive since $R_{1}$ and $R_{2}$ are positive and $B_{1}>B_{2}>B_{3}$.

A computation shows that

$$
\begin{aligned}
\rho_{1} \rho_{2}-\rho_{3}= & \left(R_{1}+R_{2}\right)\left(R_{1} R_{2}\right)+\frac{\omega^{2}}{A_{1} A_{2}} J_{2}^{2} J_{1}^{1} \\
& -\frac{\omega^{2}}{A_{1} A_{2}}\left\{\left(B_{1}-B_{3}\right) R_{2} J_{2}^{2}+\left(B_{2}-B_{3}\right) J_{1}^{1} R_{1}\right\} .
\end{aligned}
$$

The first two terms are small by assumption and hence $\rho_{1} \rho_{2}-\rho_{3}$ is negative. It then follows that

$$
\frac{\rho_{3}\left(\rho_{1} \rho_{2}-\rho_{3}\right)-\rho_{1} \rho_{4}}{\rho_{1} \rho_{2}-\rho_{3}}
$$

is positive.

Hence the Routh-Hurwitz sign sequence is $\{+,+,-,+,+\}$ and thus the addition of dissipation has indeed moved two eigenvalues into the right half plane, causing a linear instability.

Example 2 (Double Spherical Pendulum) In Marsden and Scheurle [1993] the double spherical pendulum is discussed. In particular, relative equilibria, called "cowboy solutions" are found explicitly and have a shape in which the horizontal 
projections of the two rods point in opposite directions. The group in this case is $S^{1}$, corresponding to rotations about the vertical axis. It is verified that indeed the linearized equations are in our standard form $M \ddot{q}+S \dot{q}+\Lambda q=0$, but where the $3 \times 3$ matrices $M, S$ and $\Lambda$ have extra zeros due to discrete symmetries. It is found that in large regions of parameter space (determined by the pendulum lengths, masses and angular momentum), that $\Lambda$ has signature $(+,-,-)$, while the eigenvalues of the linearized system lie on the imaginary axis. It follows from Theorem $\mathbf{1 . 1}$ or $\mathbf{4 . 2}$ that if one adds joint friction (so that the total angular momentum is still conserved) then the cowboy solutions become spectrally unstable. This example is a good one in that direct analytical computation of eigenvalue movement to see this instability would be quite complicated. We also point out that experiments of John Baillieul (Boston University) confirm this instability.

We also point out that similar eigenvalue and energetic situations arise in a number of other examples; among them are:

1. The heavy top - see Lewis, Ratiu, Simo and Marsden [1992]

2. The rotating liquid drop- see Lewis [1989]

3. Shear flow in a stratified fluid with Richardson number between $1 / 4$ and 1 ; see Abarbanel et al. [1986]

4. Plasma dynamics; see Morrison and Kotschenreuther [1989], Kandrup [1991], and Kandrup and Morrison [1992].

The last three examples mentioned are infinite dimensional, which provide motivation for extending our methods to cover such cases. One infinite dimensional example we can handle is the next one.

Example 3 We now consider a partial differential equation for which one can analyze dissipation induced instability by finite-dimensional techniques. We consider a Lagrangian for a model of a nonplanar rotating beam with "square" cross-section. The beam is assumed to be of Euler-Bernoulli type. It is fixed to the center of a circular plate rotating with constant angular velocity $\omega$, with undeflected position perpendicular to the plate along the $z$-axis of a Cartesian coordinate system fixed in the plate. The beam is inextensible and can deflect in the $x$ - and $y$-directions. (The planar version of this model is analyzed in Baillieul and Levi [1987].) The Lagrangian is chosen to be

$$
\begin{aligned}
L\left(x, y, x_{t}, y_{t}\right)= & \frac{1}{2} \int_{0}^{1}\left(\left(x_{t}-y \omega\right)^{2}+\left(y_{t}+x \omega\right)^{2}\right) d z \\
& -\frac{1}{2} \int_{0}^{1} k\left(y_{z z}^{2}+x_{z z}^{2}\right) d z .
\end{aligned}
$$

where $k$ is an elastic constant. 
The equations of motion with Rayleigh damping and damping constant $\gamma$ are:

$$
\begin{aligned}
& x_{t t}-2 \omega y_{t}-\omega^{2} x+k x_{z z z z}+\gamma x_{t}=0 \\
& y_{t t}+2 \omega x_{t}-\omega^{2} y+k y_{z z z z}+\gamma y_{t}=0 .
\end{aligned}
$$

The natural boundary conditions are:

$$
\begin{aligned}
& x(0)=x^{\prime}(0)=x^{\prime \prime}(1)=x^{\prime \prime \prime}(1)=0 \\
& y(0)=y^{\prime}(0)=y^{\prime \prime}(1)=y^{\prime \prime \prime}(1)=0
\end{aligned}
$$

where ' denotes the $z$-derivative.

The equilibrium states are given by $x_{t t}=y_{t t}=0, x_{t}=y_{t}=0$ and hence

$$
\begin{aligned}
& -\omega^{2} x+k x_{z z z z}=0 \\
& -\omega^{2} y+k y_{z z z z}=0 .
\end{aligned}
$$

We set $k=1$ for convenience.

The fourth order operator with the given boundary conditions has compact inverse and hence (see e.g. Baillieul and Levi [1987]) the eigenvalues of equations (6.9) are given by $0 \leq \omega_{1}^{2} \leq \omega_{2}^{2} \leq \ldots \rightarrow \infty$ with corresponding eigenfunctions $x(z)=x_{i}(z)$ and $y(z)=y_{i}(z)$ respectively. By our choice of the elastic constants, $x_{i}(z)=y_{i}(z)$.

Consider now the undamped case, $\gamma=0$, and write the equations in first order form, letting $q_{1}=x, q_{2}=y, p_{1}=x_{t}, p_{2}=y_{t}$. We obtain:

$$
\begin{aligned}
& q_{1 t}=p_{1} \\
& q_{2 t}=p_{2} \\
& p_{1 t}=2 \omega p_{2}+\omega^{2} q_{1}-q_{1 z z z z} \\
& p_{2 t}=-2 \omega p_{1}+\omega^{2} q_{2}-q_{2 z z z z} .
\end{aligned}
$$

Let $z=\left[q_{1} q_{2} p_{1} p_{2}\right]^{T}$. The system is thus of the form $z_{t}=A z$ where

$$
A=\left[\begin{array}{cccc}
0 & 0 & 1 & 0 \\
0 & 0 & 0 & 1 \\
-\partial^{4}+\omega^{2} & 0 & 0 & 2 \omega \\
0 & -\partial^{4}+\omega^{2} & -2 \omega & 0
\end{array}\right]
$$

The stability of the equilibria are determined by the eigenvalues of $A$. In addition to the zero eigenvalue, one can check that $A$ has eigenvalues $\pm i\left(\omega \pm \omega_{j}\right)$ with corresponding eigenvectors

$$
\begin{array}{ll}
{\left[\begin{array}{l}
x_{j} \\
i x_{j} \\
i\left(\omega+\omega_{j}\right) x_{j} \\
-i\left(\omega+\omega_{j}\right) x_{j}
\end{array}\right]} & {\left[\begin{array}{l}
x_{j} \\
-i x_{j} \\
-i\left(\omega+\omega_{j}\right) x_{j} \\
-\left(\omega+\omega_{j}\right) x_{j}
\end{array}\right]} \\
{\left[\begin{array}{l}
x_{j} \\
i x_{j} \\
i\left(\omega_{j}-\omega\right) x_{j} \\
-\left(\omega_{j}-\omega\right) x_{j}
\end{array}\right]} & {\left[\begin{array}{l}
x_{j} \\
-i x_{j} \\
-i\left(\omega_{j}-\omega\right) x_{j} \\
-\left(\omega_{j}-\omega\right) x_{j}
\end{array}\right] .}
\end{array}
$$


Now project the system onto the invariant subspace spanned by the four eigenvectors corresponding to the eigenvalues $\pm i\left(\omega \pm \omega_{j}\right)$. We see that on this subspace we have a gyroscopic system in Chetaev normal form (1.9). In fact, it is identical to the system describing the rotating bead given in $\S \mathbf{1}$, with spring constant $k=w_{j}^{2}$. Hence for $w^{2}>w_{1}^{2}$ we can see that addition of dissipation causes the system to become spectrally unstable. In fact, for $w_{j}^{2}<w^{2}<w_{j}^{2}+1$ there are $j$ gyroscopically stable Chetaev subsystems whose eigenvalues will be driven into the right-half-plane on the addition of dissipation.

\section{References}

Abarbanel, H.D.I., D.D. Holm, J.E. Marsden and T.S. Ratiu [1986] Nonlinear stability analysis of stratified fluid equilibria, Phil. Trans. R. Soc. Lond. A 318, 349-409; also Phys. Rev. Lett. 52 [1984] 2352-2355.

Abraham, R. and J.E. Marsden [1978] Foundations of Mechanics. Second Edition, Addison-Wesley Publishing Co., Reading, Mass..

Abraham, R., J.E. Marsden and T.S. Ratiu [1988] Manifolds, Tensor Analysis, and Applications. Second Edition, Springer-Verlag, New York.

Arnold, V. [1987] Dynamical Systems III. Encyclopedia of Mathematics, SpringerVerlag.

Baillieul, J. and M. Levi [1987] Rotational elastic dynamics, Physica D 27, 43-62.

Baillieul, J. and M. Levi [1991] Constrained relative motions in rotational mechanics, Arch. Rat. Mech. An. 115, 101-135.

Bellman, R. [1963] Matrix Analysis. Academic Press, New York.

Bloch, A.M., P.S. Krishnaprasad, J.E. Marsden and T.S. Ratiu [1991] Asymptotic stability, instability, and stabilization of relative equilibria, Proc. of $A C C$., Boston IEEE, 1120-1125.

Bloch, A.M., P.S. Krishnaprasad, J.E. Marsden and G. Sánchez de Alvarez [1992] Stabilization of rigid body dynamics by internal and external torques, Automatica 28, 745-756.

Brockett, R. [1970] Finite Dimensional Linear Systems. Wiley.

Chandrasekhar, K. [1977] Ellipsoidal Figures of Equilibrium. Dover.

Chetaev, N.G. [1961] The stability of Motion. Trans. by M. Nadler, Pergamon Press, New York.

Gantmacher, F.R. [1959] Theory of Matrices. Chelsea, N.Y.

Guckenheimer, J. and A. Mahalov [1992], Instability induced by symmetry reduction, Phys. Rev. Lett., 68, 2257-2260. 
Hahn, W. [1967] Stability of Motion. Springer-Verlag, New York.

Haller, G. [1992] Gyroscopic stability and its loss in systems with two essential coordinates, Int. J. Nonlinear Mech. 27, 113-127.

Holm, D.D., J.E. Marsden, T.S. Ratiu and A. Weinstein [1985] Nonlinear stability of fluid and plasma equilibria, Phys. Rep. 123, 1-116.

Kandrup, H.E. [1991] The secular instability of axisymmetric collisionless star cluster, Astrophy. J. 380, 511-514.

Kandrup, H.E. and P. Morrison [1992] Hamiltonian structure of the Vlasov-Einstein system and the problem of stability for spherical relativistic star clusters, preprint..

Kirk, V., J. E. Marsden and M. Silber [1996], Branches of stable three-tori using Hamiltonian methods in Hopf bifurcation on a rhombic lattice, Dyn. and Stab. of Systems, 11, 267-302.

Krein, M.G. [1950] A generalization of some investigations of linear differential equations with periodic coefficients. Doklady Akad. Nauk SSSR N.S. 73, 445-448.

Krishnaprasad, P.S. [1985] Lie-Poisson structures, dual-spin spacecraft and asymptotic stability, Nonl. An. Th. Meth. and Appl. 9, 1011-1035.

Krishnaprasad, P.S. and J.E. Marsden [1987] Hamiltonian structure and stability for rigid bodies with flexible attachments, Arch. Rat. Mech. An. 98, 137-158.

LaSalle, J.P. and S. Lefschetz [1963] Stability by Lyapunov's direct method. Academic Press, New York.

Levi, M. [1977] Stability of linear Hamiltonian systems with periodic coefficients. Research Report RC 6610(\#28482), IBM F.J. Watson Research Center.

Lewis, D.K. [1989] Nonlinear stability of a rotating planar liquid drop, Arch. Rat. Mech. Anal. 106, 287-333.

Lewis, D.K. [1992] Lagrangian block diagonalization. Dyn. Diff. Eqn's. 4 1-42.

Lewis, D.K. [1993] Linearized dynamics of symmetric Lagrangian systems, to appear in International Conference of Hamiltonian Dynamical Systems, Cincinnati, March 25-28, 1992.

Lewis, D.K. and T.S. Ratiu [1993] (unpublished manuscript).

Lewis, D., T.S. Ratiu, J.C. Simo and J.E. Marsden [1992] The heavy top, a geometric treatment, Nonlinearity 5, 1-48.

Lewis, D.K. and J.C. Simo [1990] Nonlinear stability of rotating pseudo-rigid bodies, Proc. Roy. Soc. Lon. A 427, 281-319. 
MacKay, R. [1991] Movement of eigenvalues of Hamiltonian equilibria under nonHamiltonian perturbation, Phys. Lett. A155, 266-268.

Marsden, J. E. and J. Scheurle [1993], Lagrangian reduction and the double spherical pendulum, $Z A M P, \mathbf{4 4}, 17-43$.

Marsden, J.E., J.C. Simo, D.K. Lewis and T.A. Posbergh [1989] A block diagonalization theorem in the energy momentum method, Cont. Math. AMS 97, 297-313.

Morrison, P.J. and M. Kotschenreuther [1989] The free energy principle, negative energy modes and stability, Proc 4th Int. Workshop on Nonlinear and Turbulent Processes in Physics, World Scientific Press.

Oh, Y.G., N. Sreenath, P.S. Krishnaprasad and J.E. Marsden [1989] The Dynamics of Coupled Planar Rigid Bodies Part 2: Bifurcations, Periodic Solutions, and Chaos, Dynamics and Diff. Eq'ns. 1, 269-298.

O'reilly, O., N. K. Malhotra and N. S. Namamchchivaya [1996], Some aspects of destabilization in reversible dynamical systems with application to follower forces, Nonlinear Dynamics, 10, 63-87.

Oh, Y.-G. [1987] A stability criterion for Hamiltonian systems with symmetry, J. Geom. Phys. 4, 163-182.

Poincaré, H. [1885] Sur l'équilibre d'une masse fluide animée d'un mouvement de rotation, Acta. Math. 7, 259.

Poincaré, H. [1892] Les formes d'équilibre d'une masse fluide en rotation, Revue Générale des Sciences 3, 809-815.

Poincaré, H. [1901] Sur la stabilité de l'équilibre des figures piriformes affectées par une masse fluide en rotation, Philosophical Transactions A 198, 333-373.

Riemann, B. [1860] Untersuchungen über die Bewegung eines flüssigen gleichartigen Ellipsoides, Abh. d. Königl. Gesell. der Wiss. zu Göttingen 9, $3-36$.

Routh, E.J. [1877] Stability of a given state of motion. Reprinted in Stability of Motion, ed. A.T. Fuller, Halsted Press, New York, 1975.

Simo, J.C., D.K. Lewis and J.E. Marsden [1991] Stability of relative equilibria I: The reduced energy momentum method, Arch. Rat. Mech. Anal. 115, 15-59.

Simo, J.C., T.A. Posbergh and J.E. Marsden [1990] Stability of coupled rigid bodies and geometrically exact rods: block diagonalization and the energymomentum method, Physics Reports 193, 280-360.

Simo, J.C., T.A. Posbergh and J.E. Marsden [1991] Stability of relative equilibria II: Three dimensional elasticity, Arch. Rat. Mech. Anal. 115, 61-100. 
Sri Namachchivaya, N., S.T. Ariaratnam [1985] On the dynamic stability of gyroscopic systems, SM Archives 10, 313-355.

Taussky, O. [1961] A Generalization of a Theorem of Lyapunov SIAM J. Appl. Math 9, 640-643.

Thomson, L. and P.G. Tait [1897] Principles of Mechanics and Dynamics. Cambridge Univ. Press (reprinted by Dover Publications Inc., 1962).

van Gils, S.A., M. Krupa and W.F. Langford [1990] Hopf bifurcation with nonsemisimple 1:1 resonance, Nonlinearity 3 , 825-830.

Wang, L.S. and P.S. Krishnaprasad [1992] Gyroscopic control and stabilization, J. Nonlinear Sci. 2, 367-415.

Whittaker, E.T. [1937] A Treatise on the Analytical Dynamics of Particles and Rigid Bodies. 4th ed., Cambridge University Press. Reprinted, 1989.

Ziegler, H. [1956] On the concept of elastic stability, Adv. Appl. Mech. 4, 351-403. 